\title{
Correlation functions of Heisenberg spin chains : the Bethe ansatz approach
}

Jean - Michel MAILLET*

Laboratoire de Physique, ENS Lyon et CNRS

46 allée d'Italie, 69364 Lyon

France

E-mail: maillet@ens-lyon.fr

We review recent progress in the computation of correlation functions of integrable Heisenberg spin-1/2 chain. We describe both finite and infinite chain results. In particular, we review a method combining analytical and numerical techniques leading to the computation of the longitudinal dynamical structure factor for finite chains of large sizes and its successful comparison to neutron scattering experiments on $\mathrm{KCuF} 3$. Long distance asymptotic behavior is also discussed. Our method is based on the resolution of the quantum inverse scattering problem in the framework of the algebraic Bethe ansatz.

Bethe Ansatz: 75 years later

October 19-21 2006,

Brussels, Belgium

${ }^{*}$ Speaker. 


\section{Introduction}

Seventy five years after its discovery, Bethe famous ansatz [1] for the solution of the Heisenberg model [2] has created a wide, active and incredibly fruitful area of scientific activity in the domain of Mathematical Physics connected both to pure Mathematics and to the forefront of several problems of modern Theoretical Physics ranging from condensed matter theory to high energy physics. These success could certainly be traced back to the increasing importance of non perturbative effects in several domains of Physics and the corresponding need for exact methods that became crucial for their deep understanding. Along the years Bethe ansatz has been playing a central role in this and has been extended and generalized in various ways that enable to tackle more and more sophisticated models in low dimensions of statistical mechanics and field theory, see $[3,4,5,6,7,8]$ and references therein. In particular, the invention of the algebraic Bethe ansatz, by Faddeev, Sklyanin and Takhtajan [9, 10], merging developments from one dimensional quantum spin chains, two dimensional models of statistical mechanics and the classical inverse scattering methods of soliton theory opened a way to construct and solve new models while providing the Mathematical framework for them $[11,12,13,14]$ leading to the discovery of the theory of quantum groups $[15,16,17,18]$. New extensions, like the Sklyanin separation of variables method $[19,20,21]$ and its link to the Baxter $Q$ operator [3] will certainly continue to push the field of integrable models forward to more success in the future.

In this lecture I would like to address what is today in my opinion the main challenging problem in the theory of quantum integrable models (after computing their spectrum), namely to obtain exact and manageable representations for their correlation functions. This issue is of great importance not only from theoretical and mathematical view points but also for applications to relevant physical situations. Although several important advances have been obtained over the years, we are still looking for a general method that could give a systematic solution to this problem. The purpose of this article is to give a review of an approach to this question elaborated in $[22,23,24,25]$ and in $[26,27,28,29]$, together with a brief account of the more recent progress obtained in [30, 31, 32, 33, 34, 35, 36, 37].

In our search for a general method to compute correlation functions of quantum integrable models our strategy was to consider a simple but representative model where it is possible to develop new tools to solve this problem. Such an archetype of quantum integrable lattice models is provided by the original $X X X$ and $X X Z$ spin- $\frac{1}{2}$ Heisenberg [2] chain in a magnetic field solved by Bethe and his followers [1, 38, 39, 40, 41, 42, 3, 4, 5, 6, 7].

The $X X Z$ spin- $\frac{1}{2}$ Heisenberg chain in a magnetic field is a quantum interacting model defined on a one-dimensional lattice with Hamiltonian,

$$
\begin{aligned}
& H=H^{(0)}-h S_{z}, \\
& H^{(0)}=\sum_{m=1}^{M}\left\{\sigma_{m}^{x} \sigma_{m+1}^{x}+\sigma_{m}^{y} \sigma_{m+1}^{y}+\Delta\left(\sigma_{m}^{z} \sigma_{m+1}^{z}-1\right)\right\}, \\
& S_{z}=\frac{1}{2} \sum_{m=1}^{M} \sigma_{m}^{z}, \quad\left[H^{(0)}, S_{z}\right]=0 .
\end{aligned}
$$


Here $\Delta$ is the anisotropy parameter, $h$ denotes the magnetic field, and $\sigma_{m}^{x, y, z}$ are the local spin operators (in the spin- $\frac{1}{2}$ representation) associated with each site $m$ of the chain. The quantum space of states is $\mathscr{H}=\otimes_{m=1}^{M} \mathscr{H}_{m}$, where $\mathscr{H}_{m} \sim \mathbb{C}^{2}$ is called local quantum space, with $\operatorname{dim} \mathscr{H}=2^{M}$. The operators $\sigma_{m}^{x, y, z}$ act as the corresponding Pauli matrices in the space $\mathscr{H}_{m}$ and as the identity operator elsewhere. For simplicity, the length of the chain $M$ is chosen to be even and we assume periodic boundary conditions; note however that the open boundary conditions case can be considered as well $[73,74]$. Since the simultaneous reversal of all spins is equivalent to a change of sign of the magnetic field, it is enough to consider the case $h \geq 0$.

The first task to solve such a model is to describe the spectrum of its Hamiltonian. The method to compute eigenvectors and associated energy levels of the Heisenberg spin chains goes back to Bethe in 1931 [1, 39, 40, 41, 42] and is known as the Bethe ansatz. An algebraic version of it has been invented in the late 70's by Faddeev, Sklyanin and Taktajan $[9,10]$.

The next fundamental problem is to compute matrix elements of spin operators $\sigma_{m}^{x, y, z}$ in the eigenvectors basis of $H$ and then all correlation functions of spin operators : at zero temperature they reduce to the average value of products of spin operators in the lowest energy level state (the ground state). Let us denote by $\left|\psi_{g}\right\rangle$ the normalized ground state vector. Let $E_{m}^{\varepsilon_{m}^{\prime}, \varepsilon_{m}}$ be the elementary operators acting at site $m$ as the $2 \times 2$ matrices $E_{l k}^{\varepsilon^{\prime}, \varepsilon}=\delta_{l, \varepsilon^{\prime}} \delta_{k, \varepsilon}$. Any $n$-point correlation function can be reconstructed as a sum of the following elementary blocks,

$$
F_{m}\left(\left\{\varepsilon_{j}, \varepsilon_{j}^{\prime}\right\}, h\right)=\left\langle\psi_{g}\left|\prod_{j=1}^{m} E_{j}^{\varepsilon_{j}^{\prime}, \varepsilon_{j}}\right| \psi_{g}\right\rangle .
$$

The knowledge of such correlation functions was for a long time restricted to the free fermion point $\Delta=0$, a case for which nevertheless tremendous works have been necessary to obtain full answers [43, 44, 45, 46, 47, 48, 49, 50, 51]. Going beyond the free Fermion case has been a major challenge for the last twenty years.

For integrable quantum spin chains and lattice models $[3,7]$, the first attempts to go beyond free Fermion models relied on the algebraic Bethe ansatz techniques $[9,10]$ and was undertaken by Izergin and Korepin (see e.g. [52, 53, 7] and references therein). Their approach yields formulae for the correlation functions written as vacuum expectation values of some determinants depending on so-called "dual fields" which were introduced to overcome the huge combinatorial sums arising in particular from the action of local operators on Bethe states. However these formulae are not completely explicit, since these "dual fields" cannot be eliminated in a simple way from the final result.

In the last fifteen years, two main approaches to a more explicit computation of form factors and correlation functions have been developed, mainly for lattice models.

One of these approaches was initiated by Jimbo, Miwa and their collaborators $[54,8]$ and enables, using some hypothesis, to compute form factors and correlation functions of quantum spin chains of infinite length (and in their massive regime) by expressing them in terms of traces of $q$ deformed vertex operators over an irreducible highest weight representation of the corresponding quantum affine algebra. These traces turn out to satisfy an axiomatic system of equations called $q$-deformed Knizhnik-Zamolodchikov ( $q$-KZ) equations, the solutions of which can be expressed in terms of multiple integral formulae. Using these equations similar formulae can be conjectured in the massless regime [55]. Recently, a more algebraic representation for the solution of these 
$q$-deformed Knizhnik-Zamolodchikov equations have been obtained for the XXX and XXZ (and conjectured for the XYZ) spin 1/2 chains; in these representations, all elementary blocks of the correlation functions can be expressed in terms of some transcendental functions [56, 57, 58, 59]. A detailed review of the approach can be found in [58]. The most recent advances along these lines are described in this workshop in the seminars of Miwa and Smirnov.

These results together with their extension to non-zero magnetic field have been obtained in 1999 [23, 24] using the algebraic Bethe ansatz framework $[9,10,6]$ and the actual resolution of the so-called quantum inverse scattering problem [23, 25]. At zero magnetic field it gives a complete proof of the multiple integral representations obtained in $[54,8,55]$ both for massive and massless regimes. Hence, together with the works [54, 8, 55], it also gives a proof that correlation functions of the XXZ (inhomogeneous) chain indeed satisfy (reduced) $q$-deformed Knizhnik-Zamolodchikov equations, an essential ingredient in the promising approach presented in this workshop by Miwa and Smirnov. Moreover, time or temperature dependent correlation functions can also be computed [30,31,60] using such techniques.

This method allows also for the computation of the matrix elements of the local spin operators and the above elementary blocks of the correlation functions for the finite chain. Hence, thermodynamic limit can be considered separately. In particular, using both analytical results from Bethe ansatz for these matrix elements of the spin operators [22, 23, 24, 25] and numerical methods to take the summation over intermediate states it has been possible recently to compute $[35,36] \mathrm{dy}$ namical structure factors (i.e., Fourier transform of the dynamical spin-spin correlation functions) for finite $X X Z$ Heisenberg spin chain in a magnetic field (with for example 500 or 1000 sites) and to compare successfully these theoretical results with actual neutron scattering experiments, for example on $\mathrm{KCuF} 3$. As motivation for the method to be presented in this review article, I would like to give here this comparison :

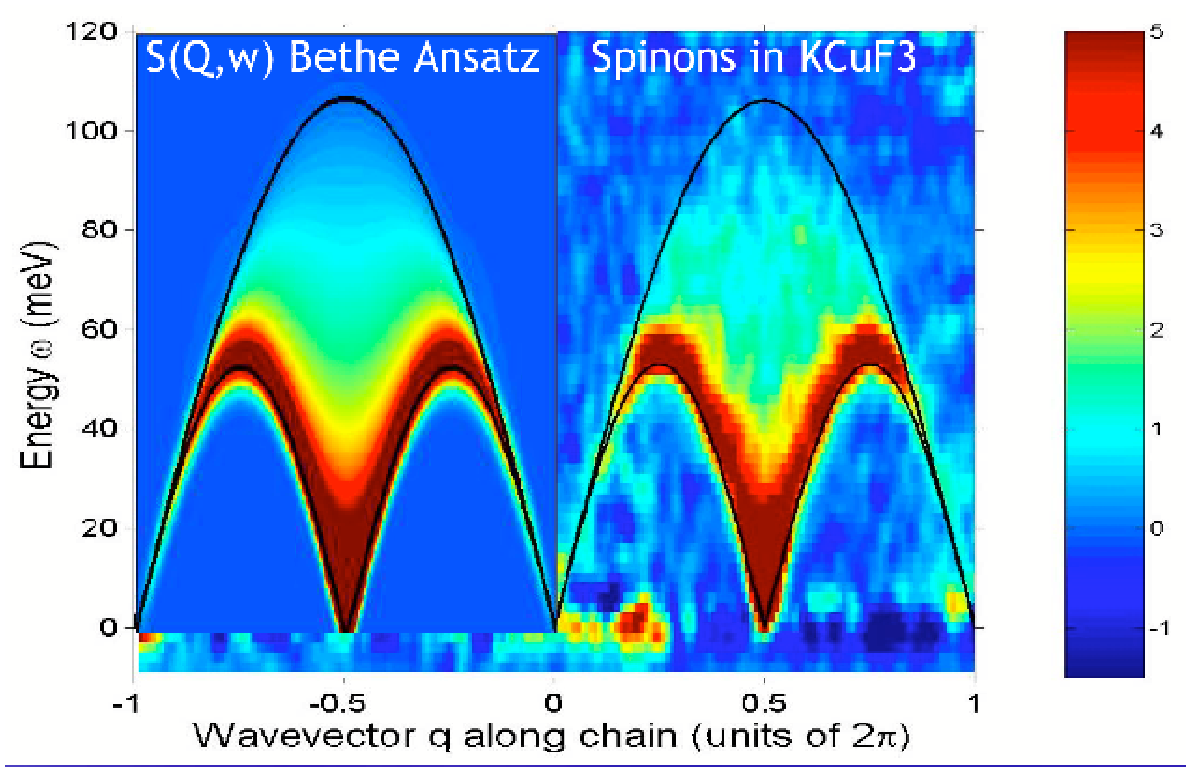

Here, the function $S(q, \omega)$ is the Fourier transform of the dynamical spin-spin correlation function. The Bethe ansatz curve (on the left) is computed here for a chain of 500 sites while the experimental curve obtained by A. Tennant and his team [61] by inelastic neutron scattering experiments on $\mathrm{KCuF} 3$ is presented on the right. Colors indicate the height of the function $S(q, \omega)$ (picture and experimental data courtesy A. Tennant). 
This article is meant to be a rather brief review of the problem of correlation functions. More detailed account of the results sketched here together with their proofs can be found in the original articles $[22,23,24,25,26,27,28,29,30,31,32]$ and in $[33,34,35,36,37]$.

This article organized as follows. The space of states of the Heisenberg spin chain will be described in the next section. It includes a brief introduction to the algebraic Bethe ansatz and to various tools of importance in the computation of correlation functions, like in particular the solution of the quantum inverse scattering problem and the determinant representations of the scalar products of states. Section 3 is devoted to the correlation functions of the finite chain. In particular we describe the method leading to the computation of the dynamical structure factor presented in the above figure. Correlation functions in the thermodynamic limit are studied in the section 4. In the section 5 we describe several exact and asymptotic results together with some open problems. Conclusions and perspectives are given in the last section.

\section{The space of states : algebraic Bethe ansatz}

The space of states is of dimension $2^{M}$. As can be observed from the definition of the Hamiltonian in (1.1), the construction of its eigenvectors is rather non trivial. The purpose of this section is to briefly explain the basics of the knowledge of the space of states in the framework of the algebraic Bethe ansatz, leading in particular to the determination of the spectrum of (1.1).

\subsection{Algebraic Bethe ansatz}

The algebraic Bethe ansatz originated from the fusion of the original (coordinate) Bethe ansatz and of the inverse scattering method in its Hamiltonian formulation $[9,10,6]$. At the root of the algebraic Bethe ansatz method is the construction of the quantum monodromy matrix. In the case of the $X X Z$ chain (1.1) the monodromy matrix is a $2 \times 2$ matrix,

$$
T(\lambda)=\left(\begin{array}{ll}
A(\lambda) & B(\lambda) \\
C(\lambda) & D(\lambda)
\end{array}\right)
$$

with operator-valued entries $A, B, C$ and $D$ which depend on a complex parameter $\lambda$ (spectral parameter) and act in the quantum space of states $\mathscr{H}$ of the chain. One of the main property of these operators is that the trace of $T$, namely $A+D$, commutes with the Hamiltonian $H$, while operators $B$ and $C$ can be used as creation operators of respectively eigenvectors and dual eigenvectors of $A+D$ and hence of $H$ itself. The monodromy matrix is defined as the following ordered product,

$$
T(\lambda)=L_{M}(\lambda) \ldots L_{2}(\lambda) L_{1}(\lambda),
$$

where $L_{n}(\lambda)$ denotes the quantum $L$-operator at the site $n$ of the chain:

$$
L_{n}(\lambda)=\left(\begin{array}{cc}
\sinh \left(\lambda+\frac{\eta}{2} \sigma_{n}^{z}\right) & \sinh \eta \sigma_{n}^{-} \\
\sinh \eta \sigma_{n}^{+} & \sinh \left(\lambda-\frac{\eta}{2} \sigma_{n}^{z}\right)
\end{array}\right) .
$$

The parameter $\eta$ is related to the anisotropy parameter as $\Delta=\cosh \eta$. It follows from this definition that the monodromy matrix is an highly non local operator in terms of the local spin operators 
$\sigma_{n}^{x, y, z}$. However, the commutation relations between the operators $A, B, C, D$ can be computed in a simple way. They are given by the quantum $R$-matrix,

$$
R(\lambda, \mu)=\left(\begin{array}{cccc}
1 & 0 & 0 & 0 \\
0 & b(\lambda, \mu) & c(\lambda, \mu) & 0 \\
0 & c(\lambda, \mu) & b(\lambda, \mu) & 0 \\
0 & 0 & 0 & 1
\end{array}\right)
$$

where

$$
b(\lambda, \mu)=\frac{\sinh (\lambda-\mu)}{\sinh (\lambda-\mu+\eta)}, c(\lambda, \mu)=\frac{\sinh (\eta)}{\sinh (\lambda-\mu+\eta)},
$$

The $R$-matrix is a linear operator in the tensor product $V_{1} \otimes V_{2}$, where each $V_{i}$ is isomorphic to $\mathbf{C}^{2}$, and depends generically on two spectral parameters $\lambda_{1}$ and $\lambda_{2}$ associated to these two vector spaces. It is denoted by $R_{12}\left(\lambda_{1}, \lambda_{2}\right)$. Such an $R$-matrix satisfies the Yang-Baxter equation,

$$
R_{12}\left(\lambda_{1}, \lambda_{2}\right) R_{13}\left(\lambda_{1}, \lambda_{3}\right) R_{23}\left(\lambda_{2}, \lambda_{3}\right)=R_{23}\left(\lambda_{2}, \lambda_{3}\right) R_{13}\left(\lambda_{1}, \lambda_{3}\right) R_{12}\left(\lambda_{1}, \lambda_{2}\right) \text {. }
$$

It gives the following commutation relations among the operators entries of the monodromy matrix,

$$
R_{12}(\lambda, \mu) T_{1}(\lambda) T_{2}(\mu)=T_{2}(\mu) T_{1}(\lambda) R_{12}(\lambda, \mu),
$$

with the tensor notations $T_{1}(\lambda)=T(\lambda) \otimes \operatorname{Id}$ and $T_{2}(\mu)=\operatorname{Id} \otimes T(\mu)$. These commutation relations imply in particular that the transfer matrices, defined as

$$
\mathscr{T}(\lambda)=\operatorname{tr} T(\lambda)=A(\lambda)+D(\lambda),
$$

commute for different values of the spectral parameter $[\mathscr{T}(\lambda), \mathscr{T}(\mu)]=0$ and also with $S_{z}$, $\left[\mathscr{T}(\lambda), S_{z}\right]=0$. The Hamiltonian (1.2) at $h=0$ is related to $\mathscr{T}(\lambda)$ by the 'trace identity'

$$
H^{(0)}=2 \sinh \eta \frac{d \mathscr{T}(\lambda)}{d \lambda} \mathscr{T}^{-1}(\lambda)_{\left.\right|_{\lambda=\frac{\eta}{2}}}-2 M \cosh \eta .
$$

Therefore, the spectrum of the Hamiltonian (1.1) is given by the common eigenvectors of the transfer matrices and of $S_{z}$.

For technical reasons, it is actually convenient to introduce a slightly more general object, the twisted transfer matrix

$$
\mathscr{T}_{\kappa}(\lambda)=A(\lambda)+\kappa D(\lambda)
$$

where $\kappa$ is a complex parameter. The particular case of $\mathscr{T}_{\kappa}(\lambda)$ at $\kappa=1$ corresponds to the usual (untwisted) transfer matrix $\mathscr{T}(\lambda)$. It will be also convenient to consider an inhomogeneous version of the $X X Z$ chain, for which

$$
T_{1 \ldots M}\left(\lambda ; \xi_{1}, \ldots, \xi_{M}\right)=L_{M}\left(\lambda-\xi_{M}+\eta / 2\right) \ldots L_{1}\left(\lambda-\xi_{1}+\eta / 2\right) .
$$

Here, $\xi_{1}, \ldots, \xi_{M}$ are complex parameters (inhomogeneity parameters) attached to each site of the lattice. The homogeneous model (1.1) corresponds to the case where $\xi_{j}=\eta / 2$ for $j=1, \ldots, M$. 
In the framework of algebraic Bethe ansatz, an arbitrary quantum state can be obtained from the vectors generated by multiple action of operators $B(\lambda)$ on the reference vector $|0\rangle$ with all spins up (respectively by multiple action of operators $C(\lambda)$ on the dual reference vector $\langle 0|$ ),

$$
|\psi\rangle=\prod_{j=1}^{N} B\left(\lambda_{j}\right)|0\rangle, \quad\langle\psi|=\langle 0| \prod_{j=1}^{N} C\left(\lambda_{j}\right), \quad N=0,1, \ldots, M .
$$

\subsection{Description of the spectrum}

Let us consider here the subspace $\mathscr{H}^{(M / 2-N)}$ of the space of states $\mathscr{H}$ with a fixed number $N$ of spins down. In this subspace, the eigenvectors $\left|\psi_{\kappa}(\{\lambda\})\right\rangle$ (respectively $\left\langle\psi_{\kappa}(\{\lambda\})\right|$ ) of the twisted transfer matrix $\mathscr{T}_{\kappa}(\mu)$ can be constructed in the form (2.12), where the parameters $\lambda_{1}, \ldots, \lambda_{N}$ satisfy the system of twisted Bethe equations

$$
\mathscr{Y}_{\kappa}\left(\lambda_{j} \mid\{\lambda\}\right)=0, \quad j=1, \ldots, N .
$$

Here, the function $\mathscr{Y}_{\kappa}$ is defined as

$$
\mathscr{Y}_{\kappa}(\mu \mid\{\lambda\})=a(\mu) \prod_{k=1}^{N} \sinh \left(\lambda_{k}-\mu+\eta\right)+\kappa d(\mu) \prod_{k=1}^{N} \sinh \left(\lambda_{k}-\mu-\eta\right)
$$

and $a(\lambda), d(\lambda)$ are the eigenvalues of the operators $A(\lambda)$ and $D(\lambda)$ on the reference state $|0\rangle$. In the normalization (2.3) and for the inhomogeneous model (2.11), we have

$$
a(\lambda)=\prod_{a=1}^{M} \sinh \left(\lambda-\xi_{a}+\eta\right), \quad d(\lambda)=\prod_{a=1}^{M} \sinh \left(\lambda-\xi_{a}\right)
$$

The corresponding eigenvalue of $\mathscr{T}_{\kappa}(\mu)$ on $\left|\psi_{\kappa}(\{\lambda\})\right\rangle$ (or on a dual eigenvector) is

$$
\tau_{\kappa}(\mu \mid\{\lambda\})=a(\mu) \prod_{k=1}^{N} \frac{\sinh \left(\lambda_{k}-\mu+\eta\right)}{\sinh \left(\lambda_{k}-\mu\right)}+\kappa d(\mu) \prod_{k=1}^{N} \frac{\sinh \left(\mu-\lambda_{k}+\eta\right)}{\sinh \left(\mu-\lambda_{k}\right)} .
$$

The solutions of the system of twisted Bethe equations (2.13) have been analyzed in [62]. In general, not all of these solutions correspond to eigenvectors of $\mathscr{T}_{\kappa}(\mu)$.

DEFINITION 2.1. A solution $\{\lambda\}$ of the system (2.13) is called admissible if

$$
d\left(\lambda_{j}\right) \prod_{\substack{k=1 \\ k \neq j}}^{N} \sinh \left(\lambda_{j}-\lambda_{k}+\eta\right) \neq 0, \quad j=1, \ldots, N
$$

and un-admissible otherwise. A solution is called off-diagonal if the corresponding parameters $\lambda_{1}, \ldots, \lambda_{N}$ are pairwise distinct, and diagonal otherwise.

One of the main result of [62] is that, for generic parameters $\kappa$ and $\{\xi\}$, the set of the eigenvectors corresponding to the admissible off-diagonal solutions of the system of twisted Bethe equations (2.13) form a basis in the subspace $\mathscr{H}^{(M / 2-N)}$. It has been proven in [31] that this result is still valid in the homogeneous case $\xi_{j}=\eta / 2, j=1, \ldots, N$, at least if $\kappa$ is in a punctured vicinity of the origin (i.e. $0<|\kappa|<\kappa_{0}$ for $\kappa_{0}$ small enough). Note however that, for specific values of $\kappa$ 
and $\{\xi\}$, the basis of the eigenvectors in $\mathscr{H}^{(M / 2-N)}$ may include some states corresponding to un-admissible solutions of (2.13) (in particular in the homogeneous limit at $\kappa=1$ ).

At $\kappa=1$, it follows from the trace identity (2.9) that the eigenvectors of the transfer matrix coincide, in the homogeneous limit, with the ones of the Hamiltonian (1.1). The corresponding eigenvalues in the case of zero magnetic field can be obtained from (2.9), (2.16):

$$
H^{(0)}|\psi(\{\lambda\})\rangle=\left(\sum_{j=1}^{N} E\left(\lambda_{j}\right)\right) \cdot|\psi(\{\lambda\})\rangle,
$$

where the (bare) one-particle energy $E(\lambda)$ is equal to

$$
E(\lambda)=\frac{2 \sinh ^{2} \eta}{\sinh \left(\lambda+\frac{\eta}{2}\right) \sinh \left(\lambda-\frac{\eta}{2}\right)} .
$$

\subsection{Drinfel'd twist and F-basis}

As already noted, the operators $A, B, C, D$ are highly non local in terms of local spin operators. There exists however an interesting description of these operators by means of a change of basis of the space of states. In particular, this basis will provide a direct access to the scalar products of states. The root of this new basis is provided by the notion of Drinfel'd twist [18] associated to the $R$-matrix of the $X X Z$ chain. It leads to the notion of factorizing $F$-matrices. To be essentially self-contained we briefly recall here their main properties and refer to [22] for more details and proofs.

DEFINITION 2.2. For inhomogeneity parameters $\xi_{j}$ in generic positions and for any integer $n$ one can associate to any element $\sigma$ of the symmetric group $S_{n}$ of order $n$ a unique $R$-matrix $R_{1 \ldots n}^{\sigma}\left(\xi_{1}, \ldots, \xi_{n}\right)$, denoted for simplicity $R_{1 \ldots n}^{\sigma}$, constructed as an ordered product (depending on $\sigma)$ of the elementary $R$-matrices $R_{i j}\left(\xi_{i}, \xi_{j}\right)$.

We have the following property for arbitrary integer $n$ :

PROPOSITION 2.1.

$$
R_{1 \ldots n}^{\sigma} T_{1 \ldots n}\left(\lambda ; \xi_{1}, \ldots, \xi_{n}\right)=T_{\sigma(1) \ldots \sigma(n)}\left(\lambda ; \xi_{\sigma(1)}, \ldots, \xi_{\sigma(n)}\right) R_{1 \ldots n}^{\sigma} .
$$

We can now define the notion of factorizing $F$-matrix :

DEFINITION 2.3. A factorizing $F$-matrix associated to a given elementary $R$ matrix is an invertible matrix $F_{1 \ldots n}\left(\xi_{1}, \ldots, \xi_{n}\right)$, defined for arbitrary integer $n$, satisfying the following relation for any element $\sigma$ of $S_{n}$ :

$$
F_{\sigma(1) \ldots \sigma(n)}\left(\xi_{\sigma(1)}, \ldots, \xi_{\sigma(n)}\right) R_{1 \ldots n}^{\sigma}\left(\xi_{1}, \ldots, \xi_{n}\right)=F_{1 \ldots n}\left(\xi_{1}, \ldots, \xi_{n}\right) .
$$

In other words, such an $F$-matrix factorizes the corresponding $R$-matrix for arbitrary integer $n$. Taking into account the fact that the parameters $\xi_{n}$ are in one to one correspondence with the vector spaces $\mathscr{H}_{n}$, we can adopt simplified notations such that

$$
\begin{aligned}
F_{1 \ldots n}\left(\xi_{1}, \ldots, \xi_{n}\right) & =F_{1 \ldots n}, \\
F_{\sigma(1) \ldots \sigma(n)}\left(\xi_{\sigma(1)}, \ldots, \xi_{\sigma(n)}\right) & =F_{\sigma(1) \ldots \sigma(n)} .
\end{aligned}
$$


THEOREM 2.1. [22] For the XXZ model with inhomogeneity parameters $\xi_{n}$ in generic positions, there exist a factorizing, triangular $F$-matrix. It is constructed explicitly from the R-matrix.

It has two important properties :

PROPOSITION 2.2. [22] In the F-basis, the monodromy matrix $\widetilde{T}$

$$
\widetilde{T}_{1 \ldots M}\left(\lambda ; \xi_{1}, \ldots, \xi_{M}\right)=F_{1 \ldots M} T_{1 \ldots M}\left(\lambda ; \xi_{1}, \ldots, \xi_{M}\right) F_{1 \ldots M}^{-1},
$$

is totally symmetric under any simultaneous permutations of the lattice sites $i$ and of the corresponding inhomogeneity parameters $\xi_{i}$.

The second property gives the explicit expressions of the monodromy matrix in the $F$-basis. For the XXZ- $\frac{1}{2}$ model, the quantum monodromy operator is a $2 \times 2$ matrix with entries $A, B, C, D$ which are obtained as sums of $2^{M-1}$ operators which themselves are products of $M$ local spin operators on the quantum chain. As an example, the $B$ operator is given as

$$
B_{1 \ldots M}(\lambda)=\sum_{i=1}^{N} \sigma_{i}^{-} \Omega_{i}+\sum_{i \neq j \neq k} \sigma_{i}^{-}\left(\sigma_{j}^{-} \sigma_{k}^{+}\right) \Omega_{i j k}+\text { higher terms }
$$

where the matrices $\Omega_{i}, \Omega_{i j k}$, are diagonal operators acting respectively on all sites but $i$, on all sites but $i, j, k$, and the higher order terms involve more and more exchange spin terms like $\sigma_{j}^{-} \sigma_{k}^{+}$. It means that the $B$ operator returns one spin somewhere on the chain, this operation being however dressed non-locally and with non-diagonal operators by multiple exchange terms of the type $\sigma_{j}^{-} \sigma_{k}^{+}$.

So, whereas these formulas in the original basis are quite involved, their expressions in the $F$-basis simplify drastically :

Proposition 2.3. [22] The operators $D, B$ and $C$ in the $F$-basis are given by the formulas

$$
\begin{aligned}
& \widetilde{D}_{1 \ldots M}\left(\lambda ; \xi_{1}, \ldots, \xi_{M}\right)=\stackrel{M}{\otimes}\left(\begin{array}{cc}
b\left(\lambda, \xi_{i}\right) & 0 \\
0 & 1
\end{array}\right)_{[i]} . \\
& \widetilde{B}_{1 \ldots M}(\lambda)=\sum_{i=1}^{M} \sigma_{i}^{-} c\left(\lambda, \xi_{i}\right) \underset{j \neq i}{\otimes}\left(\begin{array}{cr}
b\left(\lambda, \xi_{j}\right) & 0 \\
0 & b^{-1}\left(\xi_{j}, \xi_{i}\right)
\end{array}\right)_{[j]} . \\
& \widetilde{C}_{1 \ldots M}(\lambda)=\sum_{i=1}^{M} \sigma_{i}^{+} c\left(\lambda, \xi_{i}\right) \underset{j \neq i}{\otimes}\left(\begin{array}{ccc}
b\left(\lambda, \xi_{j}\right) & b^{-1}\left(\xi_{i}, \xi_{j}\right) & 0 \\
0 & 1
\end{array}\right)_{[j]},
\end{aligned}
$$

and the operator $\widetilde{A}$ can be obtained from quantum determinant relations.

We wish first to stress that while the operators $\widetilde{A}, \widetilde{B}, \widetilde{C}, \widetilde{D}$ satisfy the same quadratic commutation relations as $A, B, C, D$, they are completely symmetric under simultaneous exchange of the inhomogeneity parameters and the of the spaces $\mathscr{H}_{n}$. It really means that the factorizing $F$-matrices we have constructed solve the combinatorial problem induced by the non-trivial action of the permutation group $S_{M}$ given by the $R$-matrix. In the $F$-basis the action of the permutation group on the operators $\widetilde{A}, \widetilde{B}, \widetilde{C}, \widetilde{D}$ is trivial. 
Further, it can be shown that the pseudo-vacuum vector is left invariant, namely, it is an eigenvector of the total $F$-matrix with eigenvalue 1; in particular, the algebraic Bethe ansatz can be carried out also in the $F$-basis. Hence, a direct computation of Bethe eigenvectors and of their scalar products in this $F$-basis is made possible, while it was a priori very involved in the original basis. There, only commutation relations between the operators $A, B, C, D$ can be used, leading (see [7]) to very intricate sums over partitions.

\subsection{Solution of the quantum inverse problem}

The very simple expressions of the monodromy matrix operators entries $D, B, C$ in the $F$ basis suggests that any local operator $E_{j}^{\varepsilon_{j}^{\prime}, \varepsilon_{j}}$, acting in a local quantum space $\mathscr{H}_{j}$ at site $j$, can be expressed in terms of the entries of the monodromy matrix. This is the so-called quantum inverse scattering problem. The solution to this problem was found in $[23,25]$ :

THEOREM 2.2.

$$
E_{j}^{\varepsilon_{j}^{\prime}, \varepsilon_{j}}=\prod_{\alpha=1}^{j-1} \mathscr{T}\left(\xi_{\alpha}\right) \cdot T_{\varepsilon_{j}, \varepsilon_{j}^{\prime}}\left(\xi_{j}\right) \cdot \prod_{\alpha=1}^{j} \mathscr{T}^{-1}\left(\xi_{\alpha}\right)
$$

The proof of this theorem is elementary (see $[23,25])$ and hence it can be obtained for a large class of lattice integrable models. It relies essentially on the property that the $R$-matrix $R(\lambda, \mu)$ reduces to the permutation operator for $\lambda=\mu$. An immediate consequence of this theorem is that the operators $A, B, C$, and $D$ generate the space of all operators acting in $\mathscr{H}$.

\subsection{Scalar products}

We give here the expressions for the scalar product of an eigenvector of the twisted transfer matrix with any arbitrary state of the form (2.12). These scalar products can be expressed as determinant of rather simple matrices. The root of all these determinants is in fact the determinant representation for the partition function of the 6-vertex model with domain wall boundary conditions [63]. Let us first define, for arbitrary positive integers $n, n^{\prime}\left(n \leq n^{\prime}\right)$ and arbitrary sets of variables $\lambda_{1}, \ldots, \lambda_{n}, \mu_{1}, \ldots, \mu_{n}$ and $v_{1}, \ldots, v_{n^{\prime}}$ such that $\{\lambda\} \subset\{v\}$, the $n \times n$ matrix $\Omega_{\kappa}(\{\lambda\},\{\mu\} \mid\{v\})$ as

$$
\begin{aligned}
\left(\Omega_{\kappa}\right)_{j k}(\{\lambda\},\{\mu\} \mid\{v\})=a\left(\mu_{k}\right) t\left(\lambda_{j}, \mu_{k}\right) & \prod_{a=1}^{n^{\prime}} \sinh \left(v_{a}-\mu_{k}+\eta\right) \\
& -\kappa d\left(\mu_{k}\right) t\left(\mu_{k}, \lambda_{j}\right) \prod_{a=1}^{n^{\prime}} \sinh \left(v_{a}-\mu_{k}-\eta\right)
\end{aligned}
$$

with

$$
t(\lambda, \mu)=\frac{\sinh \eta}{\sinh (\lambda-\mu) \sinh (\lambda-\mu+\eta)}
$$


Proposition 2.4. $[64,23,30]$ Let $\left\{\lambda_{1}, \ldots, \lambda_{N}\right\}$ be a solution of the system of twisted Bethe equations (2.13), and $\mu_{1}, \ldots, \mu_{N}$ be generic complex numbers. Then,

$$
\begin{aligned}
\left\langle 0\left|\prod_{j=1}^{N} C\left(\mu_{j}\right)\right| \psi_{\kappa}(\{\lambda\})\right\rangle & =\left\langle\psi_{\kappa}(\{\lambda\})\left|\prod_{j=1}^{N} B\left(\mu_{j}\right)\right| 0\right\rangle \\
& =\frac{\prod_{a=1}^{N} d\left(\lambda_{a}\right) \prod_{a, b=1}^{N} \sinh \left(\mu_{b}-\lambda_{a}\right)}{\prod_{a>b}^{N} \sinh \left(\lambda_{a}-\lambda_{b}\right) \sinh \left(\mu_{b}-\mu_{a}\right)} \cdot \operatorname{det}_{N}\left(\frac{\partial}{\partial \lambda_{j}} \tau_{\kappa}\left(\mu_{k} \mid\{\lambda\}\right)\right) \\
& =\frac{\prod_{a=1}^{N} d\left(\lambda_{a}\right)}{\prod_{a>b}^{N} \sinh \left(\lambda_{a}-\lambda_{b}\right) \sinh \left(\mu_{b}-\mu_{a}\right)} \cdot \operatorname{det}_{N} \Omega_{\kappa}(\{\lambda\},\{\mu\} \mid\{\lambda\}) .
\end{aligned}
$$

These equations are valid for any arbitrary complex parameter $\kappa$, in particular at $\kappa=1$. In this case we may omit the subscript $\kappa$ and denote $(\psi, \tau, \mathscr{Y}, \Omega)=\left.\left(\psi_{\kappa}, \tau_{\kappa}, \mathscr{Y}_{\kappa}, \Omega_{\kappa}\right)\right|_{\kappa=1}$. If the sets $\{\lambda\}$ and $\{\mu\}$ are different, the eigenvector $\left|\psi_{\kappa}(\{\lambda\})\right\rangle$ is orthogonal to the dual eigenvector $\left\langle\psi_{\kappa}(\{\mu\})\right|$. Otherwise we obtain a formula for the norm of the corresponding vector $[65,66,23]$,

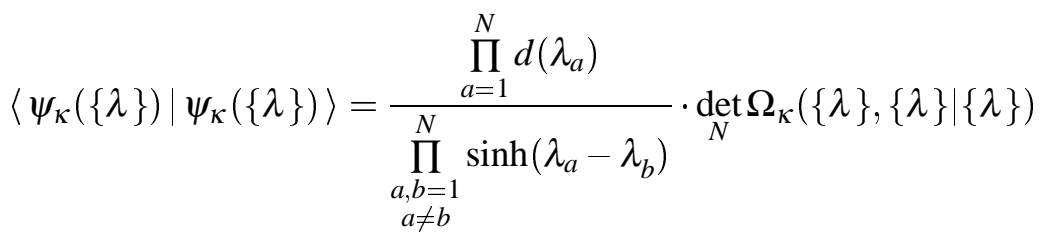

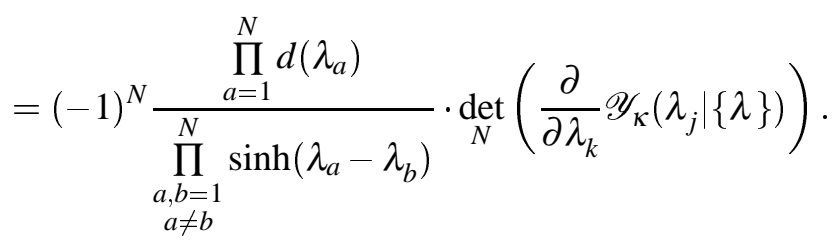

\subsection{Action of operators $A, B, C, D$ on a general state}

An important step of the computation of correlation function is to express the action of any product of local operators on any Bethe eigenvector. From the solution of the quantum inverse scattering problem, this is given by the successive action of $A, B, C, D$ operators on a vector constructed by action of $C$ operators on the reference vector. Action of $A, B, C, D$ on such a vector are well known (see for example [7]). They can be written in the following form:

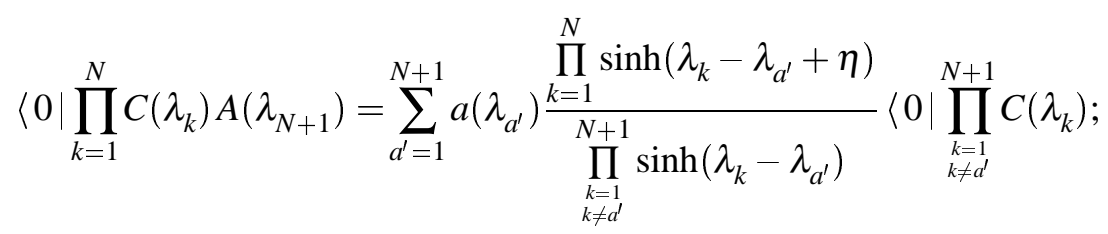

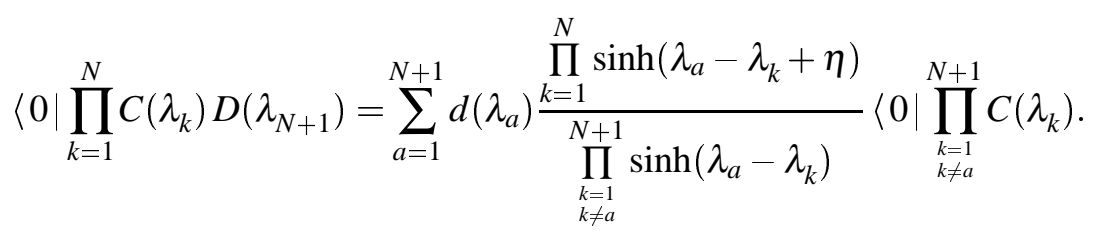


The action of the operator $B(\lambda)$ can be obtained similarly,

$$
\begin{gathered}
\langle 0| \prod_{k=1}^{N} C\left(\lambda_{k}\right) B\left(\lambda_{N+1}\right)=\sum_{a=1}^{N+1} d\left(\lambda_{a}\right) \frac{\prod_{k=1}^{N} \sinh \left(\lambda_{a}-\lambda_{k}+\eta\right)}{\prod_{\substack{k=1 \\
k \neq a}}^{N+1} \sinh \left(\lambda_{a}-\lambda_{k}\right)} \times \\
\times \sum_{\substack{a^{\prime}=1 \\
a^{\prime} \neq a}}^{N+1} \frac{a\left(\lambda_{a^{\prime}}\right)}{\sinh \left(\lambda_{N+1}-\lambda_{a^{\prime}}+\eta\right)} \frac{\prod_{\substack{j=1 \\
j \neq a}}^{N+1} \sinh \left(\lambda_{j}-\lambda_{a^{\prime}}+\eta\right)}{\prod_{\substack{j=1 \\
j \neq a, a^{\prime}}}^{N+1} \sinh \left(\lambda_{j}-\lambda_{a^{\prime}}\right)}\langle 0| \prod_{\substack{k=1 \\
k \neq a, a^{\prime}}}^{N+1} C\left(\lambda_{k}\right),
\end{gathered}
$$

and the action of $C$ is obvious.

\section{Correlation functions : finite chain}

To compute correlation functions of some product of local operators, the following successive problems have to be addressed: $(i)$ determination of the ground state $\left\langle\psi_{g}\right|$, (ii) evaluation of the action of the product of the local operators on it, and (iii) computation of the scalar product of the resulting state with $\left|\psi_{g}\right\rangle$. Using the solution of the quantum inverse scattering problem together with the explicit determinant formulas for the scalar products and the norm of the Bethe state, one sees that matrix elements of local spin operators and correlation functions can be expressed as (multiple) sums of determinants [24]. It should be stressed that this result is purely algebraic and is valid for finite chains of arbitrary length $M$.

\subsection{Matrix elements of local operators}

We begin with the calculation of the one-point functions. These results follow directly from the solution of the quantum inverse scattering problem, the above action of operators $A, B, C$ and $D$, and the determinant representation of the scalar products. We consider,

$$
F_{N}^{-}\left(m,\left\{\mu_{j}\right\},\left\{\lambda_{k}\right\}\right)=\left\langle 0\left|\prod_{j=1}^{N+1} C\left(\mu_{j}\right) \sigma_{m}^{-} \prod_{k=1}^{N} B\left(\lambda_{k}\right)\right| 0\right\rangle,
$$

and

$$
F_{N}^{+}\left(m,\left\{\lambda_{k}\right\},\left\{\mu_{j}\right\}\right)=\left\langle 0\left|\prod_{k=1}^{N} C\left(\lambda_{k}\right) \sigma_{m}^{+} \prod_{j=1}^{N+1} B\left(\mu_{j}\right)\right| 0\right\rangle,
$$

where $\left\{\lambda_{k}\right\}_{n}$ and $\left\{\mu_{j}\right\}_{n+1}$ are solutions of Bethe equations.

Proposition 3.1. For two Bethe states with spectral parameters $\left\{\lambda_{k}\right\}_{N}$ and $\left\{\mu_{j}\right\}_{N+1}$, the matrix element of the operator $\sigma_{m}^{-}$can be represented as a determinant,

$$
\begin{aligned}
F_{N}^{-}\left(m,\left\{\mu_{j}\right\},\left\{\lambda_{k}\right\}\right)=\frac{\phi_{m-1}\left(\left\{\mu_{j}\right\}\right)}{\phi_{m-1}\left(\left\{\lambda_{k}\right\}\right)} \frac{\prod_{j=1}^{N+1} \sinh \left(\mu_{j}-\xi_{m}+\eta\right)}{\prod_{k=1}^{N} \sinh \left(\lambda_{k}-\xi_{m}+\eta\right)} \\
\cdot \frac{\operatorname{det}_{N+1} H^{-}\left(m,\left\{\mu_{j}\right\},\left\{\lambda_{k}\right\}\right)}{\prod_{N+1 \geq k>j \geq 1} \sinh \left(\mu_{k}-\mu_{j}\right) \prod_{1 \leq \beta<\alpha \leq N} \sinh \left(\lambda_{\beta}-\lambda_{\alpha}\right)},
\end{aligned}
$$




$$
\phi_{m}\left(\left\{\lambda_{k}\right\}\right)=\prod_{k=1}^{N} \prod_{j=1}^{m} b^{-1}\left(\lambda_{k}, \xi_{j}\right)
$$

and the $(N+1) \times(N+1)$ matrix $H^{-}$is defined as

$$
\begin{aligned}
H_{a b}^{-}(m) & =\frac{\varphi(\eta)}{\varphi\left(\mu_{a}-\lambda_{b}\right)}\left(a\left(\lambda_{b}\right) \prod_{\substack{j=1 \\
j \neq a}}^{N+1} \varphi\left(\mu_{j}-\lambda_{b}+\eta\right)-d\left(\lambda_{b}\right) \prod_{\substack{j=1 \\
j \neq a}}^{N+1} \varphi\left(\mu_{j}-\lambda_{b}-\eta\right)\right) \\
\text { for } b & <N+1, \\
H_{a N+1}^{-}(m) & =\frac{\varphi(\eta)}{\varphi\left(\mu_{a}-\xi_{m}+\eta\right) \varphi\left(\mu_{a}-\xi_{m}\right)} .
\end{aligned}
$$

The matrix element $F_{N}^{+}\left(m,\left\{\lambda_{k}\right\},\left\{\mu_{j}\right\}\right)$ we get,

$$
F_{N}^{+}\left(m,\left\{\lambda_{k}\right\},\left\{\mu_{j}\right\}\right)=\frac{\phi_{m}\left(\lambda_{k}\right) \phi_{m-1}\left(\lambda_{k}\right)}{\phi_{m-1}\left(\mu_{j}\right) \phi_{m}\left(\mu_{j}\right)} F_{N}^{-}\left(m,\left\{\mu_{j}\right\},\left\{\lambda_{k}\right\}\right) .
$$

The matrix elements of the operator $\sigma_{m}^{z}$ between two Bethe states have been obtained similarly [23].

\subsection{Elementary blocks of correlation functions}

In this section we consider a more general case of correlation functions : the ground state mean value of any product of the local elementary $2 \times 2$ matrices $E_{l k}^{\varepsilon^{\prime}, \varepsilon}=\delta_{l, \varepsilon^{\prime}} \delta_{k, \varepsilon}$ :

$$
F_{m}\left(\left\{\varepsilon_{j}, \varepsilon_{j}^{\prime}\right\}\right)=\frac{\left\langle\psi_{g}\left|\prod_{j=1}^{m} E_{j}^{\varepsilon_{j}^{\prime}, \varepsilon_{j}}\right| \psi_{g}\right\rangle}{\left\langle\psi_{g} \mid \psi_{g}\right\rangle} .
$$

An arbitrary $n$-point correlation function can be obtained as a sum of such mean values. Using the solution of the quantum inverse scattering problem, we reduce this problem to the computation of the ground state mean value of an arbitrary ordered product of monodromy matrix elements,

$$
F_{m}\left(\left\{\varepsilon_{j}, \varepsilon_{j}^{\prime}\right\}\right)=\phi_{m}^{-1}(\{\lambda\}) \frac{\left\langle\psi_{g}\left|T_{\varepsilon_{1}, \varepsilon_{1}^{\prime}}\left(\xi_{1}\right) \ldots T_{\varepsilon_{m}, \varepsilon_{m}^{\prime}}\left(\xi_{m}\right)\right| \psi_{g}\right\rangle}{\left\langle\psi_{g} \mid \psi_{g}\right\rangle},
$$

To calculate these mean values we first describe generically the product of the monodromy matrix elements. For that purpose, one should consider the two following sets of indices, $\alpha^{+}=\{j: 1 \leq$ $\left.j \leq m, \varepsilon_{j}=1\right\}, \operatorname{card}\left(\alpha^{+}\right)=s^{\prime}, \max _{j \in \alpha^{+}}(j) \equiv j_{\max }^{\prime}, \min _{j \in \alpha^{+}}(j) \equiv j_{\min }^{\prime}$, and similarly $\alpha^{-}=\{j:$ $\left.1 \leq j \leq m, \varepsilon_{j}^{\prime}=2\right\}, \operatorname{card}\left(\alpha^{-}\right)=s, \max _{j \in \alpha^{-}}(j) \equiv j_{\max }, \min _{j \in \alpha^{-}}(j) \equiv j_{\min }$. The intersection of these two sets is not empty and corresponds to the operators $B\left(\xi_{j}\right)$. Consider now the action, $\langle 0| \prod_{k=1}^{N} C\left(\lambda_{k}\right) T_{\varepsilon_{1}, \varepsilon_{1}^{\prime}}\left(\lambda_{N+1}\right) \ldots T_{\varepsilon_{m}, \varepsilon_{m}^{\prime}}\left(\lambda_{N+m}\right)$, applying one by one the formulae (2.32)-(2.34). For all the indices $j$ from the sets $\alpha^{+}$and $\alpha^{-}$one obtains a summation on the corresponding indices $a_{j}^{\prime}$ (for $j \in \alpha^{+}$, corresponding to the action of the operators $A(\lambda)$ or $B(\lambda)$ ) or $a_{j}$ (for $j \in \alpha^{-}$, corresponding to the action of the operators $D(\lambda)$ or $B(\lambda)$ ). As the product of the monodromy matrix elements is ordered these summations are also ordered and the corresponding indices should be taken from the following sets, $\mathbf{A}_{j}=\left\{b: 1 \leq b \leq N+m, b \neq a_{k}, a_{k}^{\prime}, k<j\right\}$ and $\mathbf{A}^{\prime}{ }_{j}=\{b: 1 \leq$ 
$\left.b \leq N+m, b \neq a_{k}^{\prime}, k<j, b \neq a_{k}, k \leq j\right\}$. Thus,

$$
\begin{aligned}
& \langle 0| \prod_{k=1}^{N} C\left(\lambda_{k}\right) T_{\varepsilon_{1}, \varepsilon_{1}^{\prime}}\left(\lambda_{N+1}\right) \ldots T_{\varepsilon_{m}, \varepsilon_{m}^{\prime}}\left(\lambda_{N+m}\right)= \\
= & \sum_{\left\{a_{j}, a_{j}^{\prime}\right\}} G_{\left\{a_{j}, a_{j}^{\prime}\right\}}\left(\lambda_{1}, \ldots, \lambda_{N+m}\right)\langle 0| \prod_{b \in \mathbf{A}_{m+1}} C\left(\lambda_{b}\right)
\end{aligned}
$$

The summation is taken over the indices $a_{j}$ for $j \in \alpha^{-}$and $a_{j}^{\prime}$ for $j \in \alpha^{+}$such that $1 \leq a_{j} \leq$ $N+j, a_{j} \in \mathbf{A}_{j}, \quad 1 \leq a_{j}^{\prime} \leq N+j, a_{j}^{\prime} \in \mathbf{A}^{\prime}{ }_{j}$. The functions $G_{\left\{a_{j}, a_{j}^{\prime}\right\}}\left(\lambda_{1}, \ldots \lambda_{N+m}\right)$ can then be easily obtained from the formulae (2.32)-(2.34) taking into acount that $\lambda_{a}=\xi_{a-N}$ for $a>N$ :

$$
\begin{aligned}
G_{\left\{a_{j}, a_{j}^{\prime}\right\}}\left(\lambda_{1}, \ldots, \lambda_{N+m}\right) & =\prod_{j \in \alpha^{-}} d\left(\lambda_{a_{j}}\right) \frac{\prod_{\substack{b=1 \\
b \in \mathbf{A}_{j}}}^{N+j-1} \sinh \left(\lambda_{a_{j}}-\lambda_{b}+\eta\right)}{\prod_{\substack{b=1 \\
b \in \mathbf{A}_{j}^{\prime}}}^{N+j} \sinh \left(\lambda_{a_{j}}-\lambda_{b}\right)} \times \\
& \times \prod_{j \in \alpha^{+}} a\left(\lambda_{a_{j}^{\prime}}\right) \frac{\prod_{\substack{b=1 \\
b \in \mathbf{A}_{j}^{\prime}}}^{N+j-1} \sinh \left(\lambda_{b}-\lambda_{a_{j}^{\prime}}+\eta\right)}{\prod_{\substack{b=1 \\
b \in \mathbf{A}_{j+1}}}^{N+j} \sinh \left(\lambda_{b}-\lambda_{a_{j}^{\prime}}\right)} .
\end{aligned}
$$

Now to calculate the normalized mean value (3.9) we apply the determinant representation for the scalar product. It should be mentioned that the number of operators $C(\lambda)$ has to be equal to the number of the operators $B(\lambda)$, as otherwise the mean value is zero, and hence the total number of elements in the sets $\alpha^{+}$and $\alpha^{-}$is $s+s^{\prime}=m$. Taking into account that in (3.9), for $b>N, \lambda_{b}=\xi_{b-N}$ one has to consider the following scalar products,

$$
\frac{\left\langle 0\left|\prod_{b \in \mathbf{A}_{m+1}} C\left(\lambda_{b}\right) \prod_{k=1}^{N} B\left(\lambda_{k}\right)\right| 0\right\rangle}{\left\langle 0\left|\prod_{k=1}^{N} C\left(\lambda_{k}\right) \prod_{k=1}^{N} B\left(\lambda_{k}\right)\right| 0\right\rangle}
$$

for all the permitted values of $a_{j}, a_{j}^{\prime}$. Finally we obtain:

$$
F_{m}\left(\left\{\varepsilon_{j}, \varepsilon_{j}^{\prime}\right\}\right)=\frac{1}{\prod_{k<l} \sinh \left(\xi_{k}-\xi_{l}\right)} \sum_{\left\{a_{j}, a_{j}^{\prime}\right\}} H_{\left\{a_{j}, a_{j}^{\prime}\right\}}\left(\lambda_{1}, \ldots, \lambda_{N+m}\right),
$$

the sum being taken on the same set of indices $a_{j}, a_{j}^{\prime}$ as in (3.10). The functions $H_{\left\{a_{j}, a_{j}^{\prime}\right\}}(\{\lambda\})$ can be obtained using (3.11) and the determinant representations for the scalar products.

\subsection{Two-point functions}

The method presented in the last section is quite straightforward and gives formally the possibility to compute any correlation function. However, it has been developed for the computation of the expectation values of the monomials $T_{a_{1} b_{1}}\left(\xi_{1}\right) \cdots T_{a_{m} b_{m}}\left(\xi_{m}\right)$, leading to the evaluation of elementary building blocks, whereas the study of the two-point functions involves big sums of such 
monomials. Indeed, let us consider for example the correlation function $\left\langle\sigma_{1}^{z} \sigma_{m+1}^{z}\right\rangle$. Then, according to the solution of the inverse scattering problem (2.27), we need to calculate the expectation value

$$
\left\langle\psi(\{\lambda\})\left|(A-D)\left(\xi_{1}\right) \cdot \prod_{a=2}^{m} \mathscr{T}\left(\xi_{a}\right) \cdot(A-D)\left(\xi_{m+1}\right) \cdot \prod_{b=1}^{m+1} \mathscr{T}^{-1}\left(\xi_{b}\right)\right| \psi(\{\lambda\})\right\rangle .
$$

Since $|\psi(\{\lambda\})\rangle$ is an eigenvector, the action of $\prod_{b=1}^{m+1} \mathscr{T}^{-1}\left(\xi_{b}\right)$ on this state merely produces a numerical factor. However, it is much more complicated to evaluate the action of $\prod_{a=2}^{m} \mathscr{T}\left(\xi_{a}\right)$. Indeed, we have to act first with $(A-D)\left(\xi_{1}\right)$ on $\langle\psi(\{\lambda\})|$ (or with $(A-D)\left(\xi_{m+1}\right)$ on $|\psi(\{\lambda\})\rangle$ ), which gives a sum of states which are no longer eigenvectors of the transfer matrix, and on which the multiple action of $\mathscr{T}$ is not simple. In fact, the product $\prod_{a=2}^{m}(A+D)\left(\xi_{a}\right)$ would lead to a sum of $2^{m-1}$ elementary blocks. This is not very convenient, in particular at large distance $m$. Therefore, to obtain manageable expressions for such correlation functions, it is of great importance to develop an alternative and compact way to express the multiple action of the transfer matrix on arbitrary states or, in other words, to make an effective re-summation of the corresponding sum of the $2^{m-1}$ terms. This can be achieved in the following way :

Proposition 3.2. Let $\kappa, x_{1}, \ldots, x_{m}$ and $\mu_{1}, \ldots, \mu_{N}$ be generic parameters. Then the action of $\prod_{a=1}^{m} \mathscr{T}_{\kappa}\left(x_{a}\right)$ on a state of the form $\langle 0| \prod_{j=1}^{N} C\left(\mu_{j}\right)$ can be formally written as

$$
\begin{aligned}
\langle 0| \prod_{j=1}^{N} C\left(\mu_{j}\right) \prod_{a=1}^{m} \mathscr{T}_{\kappa}\left(x_{a}\right) & =\frac{1}{N !} \oint_{\Gamma\{x\} \cup\lceil\mu\}} \prod_{j=1}^{N} \frac{d z_{j}}{2 \pi i} \cdot \prod_{a=1}^{m} \tau_{\kappa}\left(x_{a} \mid\{z\}\right) \cdot \prod_{a=1}^{N} \frac{1}{\mathscr{Y}_{\kappa}\left(z_{a} \mid\{z\}\right)} \\
& \times \prod_{\substack{j, k=1 \\
j<k}}^{N} \frac{\sinh \left(z_{j}-z_{k}\right)}{\sinh \left(\mu_{j}-\mu_{k}\right)} \cdot \operatorname{det}_{N} \Omega_{\kappa}(\{z\},\{\mu\} \mid\{z\}) \cdot\langle 0| \prod_{j=1}^{N} C\left(z_{j}\right),
\end{aligned}
$$

where the integration contour $\Gamma\{x\} \cup \Gamma\{\mu\}$ surrounds the points ${ }^{1} x_{1}, \ldots, x_{m}$ and $\mu_{1}, \ldots, \mu_{N}$ and does not contain any other pole of the integrand.

One of the simplest applications concerns the generating function of the two-point correlation function of the third components of spin, which is defined as the normalized expectation value $\left\langle Q_{l, m}^{\kappa}\right\rangle$ of the operator

$$
Q_{l, m}^{\kappa}=\prod_{n=l}^{m}\left(\frac{1+\kappa}{2}+\frac{1-\kappa}{2} \cdot \sigma_{n}^{z}\right)=\prod_{j=1}^{l-1} \mathscr{T}\left(\xi_{j}\right) \cdot \prod_{j=l}^{m} \mathscr{T}_{\kappa}\left(\xi_{j}\right) \cdot \prod_{j=1}^{m} \mathscr{T}^{-1}\left(\xi_{j}\right),
$$

where $|\psi(\{\lambda\})\rangle$ is an eigenvector of $\mathscr{T}(\mu)$ in the subspace $\mathscr{H}^{(M / 2-N)}$. The two-point correlation function of the third components of local spins in the eigenvector $|\psi(\{\lambda\})\rangle$ can be obtained in terms of the second 'lattice derivative' and the second derivative with respect to $\kappa$ of the generating function $\left\langle Q_{l, m}^{\kappa}\right\rangle$ at $\kappa=1$ :

$$
\begin{aligned}
& \left\langle\sigma_{l}^{z} \sigma_{l+m}^{z}\right\rangle=\left\langle\sigma_{l}^{z}\right\rangle+\left\langle\sigma_{l+m}^{z}\right\rangle-1 \\
& \quad+2 \frac{\partial^{2}}{\partial \kappa^{2}}\left\langle Q_{l, l+m}^{\kappa}-Q_{l, l+m-1}^{\kappa}-Q_{l+1, l+m}^{\kappa}+Q_{l+1, l+m-1}^{\kappa}\right\rangle_{\left.\right|_{\kappa=1}} .
\end{aligned}
$$

\footnotetext{
${ }^{1}$ More precisely, for a set of complex variables $\left\{v_{1}, \ldots, v_{l}\right\}$, the notation $\Gamma\{v\}$ should be understood in the following way: $\Gamma\{v\}$ is the boundary of a set of poly-disks $\mathscr{D}_{a}(r)$ in $\mathbb{C}^{N}$, i.e. $\Gamma\{v\}=\cup_{a=1}^{l} \overline{\mathscr{D}}_{a}(r)$ with $\overline{\mathscr{D}}_{a}(r)=\left\{z \in \mathbb{C}^{N}\right.$ : $\left.\left|z_{k}-v_{a}\right|=r, \quad k=1, \ldots, N\right\}$.
} 
Due to the translational invariance of the correlation functions in the homogeneous model, we will simply consider the expectation value $\left\langle Q_{1, m}^{\kappa}\right\rangle$. For any given eigenvector, we obtain the following result:

THEOREM 3.1. Let $\{\lambda\}$ be an admissible off-diagonal solution of the system of untwisted Bethe equations, and let us consider the corresponding expectation value $\left\langle Q_{1, m}^{\kappa}\right\rangle$ in the inhomogeneous finite $X X Z$ chain. Then there exists $\kappa_{0}>0$ such that, for $|\kappa|<\kappa_{0}$, the following representations hold:

$$
\begin{aligned}
\left\langle Q_{1, m}^{\kappa}\right\rangle=\frac{1}{N !} \oint_{\Gamma\{\xi\} \cup \Gamma\{\lambda\}} \prod_{j=1}^{N} \frac{d z_{j}}{2 \pi i} \cdot \prod_{a=1}^{m} \frac{\tau_{\kappa}\left(\xi_{a} \mid\{z\}\right)}{\tau\left(\xi_{a} \mid\{\lambda\}\right)} \cdot \prod_{a=1}^{N} \frac{1}{\mathscr{Y}_{\kappa}\left(z_{a} \mid\{z\}\right)} \\
\quad \times \operatorname{det}_{N} \Omega_{\kappa}(\{z\},\{\lambda\} \mid\{z\}) \cdot \frac{\operatorname{det}_{N} \Omega(\{\lambda\},\{z\} \mid\{\lambda\})}{\operatorname{det}_{N} \Omega(\{\lambda\},\{\lambda\} \mid\{\lambda\})}
\end{aligned}
$$

The integration contours are such that the only singularities of the integrand which contribute to the integral are the points $\xi_{1}, \ldots, \xi_{m}$ and $\lambda_{1} \ldots, \lambda_{N}$.

From this result, we can extract a compact representation for the two-point function of $\sigma^{z}$ [30]. Similar expressions exists for other correlation functions of the spin operators, and in particular for the time dependent case [30,31]. Moreover, this multiple contour integral representation permits to relate two very different ways to compute two point correlation functions of the type, $g_{12}=$ $\left\langle\Psi_{g}\left|\sigma_{i}^{\alpha} \sigma_{j}^{\beta}\right| \Psi_{g}\right\rangle$, namely,

(i) to compute the action of local operators on the ground state $\sigma_{i}^{\alpha} \sigma_{j}^{\beta}\left|\Psi_{g}\right\rangle=|\tilde{\Psi}\rangle$ and then to calculate the resulting scalar product $g_{12}=\left\langle\Psi_{g} \mid \tilde{\Psi}\right\rangle$ as was explain in the previous sections.

(ii) to insert a sum over a complete set of states $\left|\Psi_{i}\right\rangle$ (for instance, a complete set of eigenvectors of the Hamiltonian) between the local operators $\sigma_{i}^{\alpha}$ and $\sigma_{j}^{\beta}$ and to obtain the representation for the correlation function as a sum over matrix elements of local operators,

$$
g_{12}=\sum_{i}\left\langle\Psi_{g}\left|\sigma_{i}^{\alpha}\right| \Psi_{i}\right\rangle \cdot\left\langle\Psi_{i}\left|\sigma_{j}^{\beta}\right| \Psi_{g}\right\rangle
$$

In fact the above representation as multiple contour integrals contains both expansions. Indeed there is two ways to evaluate the corresponding integrals : either to compute the residues in the poles inside $\Gamma$, or to compute the residues in the poles within strips of the width $i \pi$ outside $\Gamma$.

The first way leads to a representation of the correlation function $\left\langle\sigma_{1}^{z} \sigma_{m+1}^{z}\right\rangle$ in terms of the previously obtained [26] $m$-multiple sums. Evaluation of the above contour integral in terms of the poles outside the contour $\Gamma$ gives us the expansion (ii) of the correlation function (i.e. an expansion in terms of matrix elements of $\sigma^{z}$ between the ground state and all excited states). This relation holds also for the time dependent case [30, 31].

\subsection{Towards the comparison with neutron scattering experiments}

In this section, we first briefly review all elements necessary for the computation of the dynamical spin-spin correlation functions of the anisotropic Heisenberg model, following [35, 36] and leading in particular to the successful comparison with neutron scattering experiments, see the figure given in the introduction. We start by giving our notations and discussing the eigenstates in some details. The reference state is taken to be the state with all spins up, $|0\rangle=\otimes_{i=1}^{M}|\uparrow\rangle_{i}$. Since the total magnetization commutes with the Hamiltonian, the Hilbert space separates into subspaces of 
fixed magnetization, determined from the number of reversed spins $N$. We take the number of sites $M$ to be even, and $2 N \leq M$, the other sector being accessible through a change in the reference state.

Eigenstates in each subspace are completely characterized for $2 N \leq M$ by a set of rapidities $\left\{\lambda_{j}\right\}, j=1, \ldots, N$, solution to the Bethe equations

$$
\left[\frac{\sinh \left(\lambda_{j}+i \zeta / 2\right)}{\sinh \left(\lambda_{j}-i \zeta / 2\right)}\right]^{M}=\prod_{k \neq j}^{N} \frac{\sinh \left(\lambda_{j}-\lambda_{k}+i \zeta\right)}{\sinh \left(\lambda_{j}-\lambda_{k}-i \zeta\right)}, \quad j=1, \ldots, N
$$

where $\Delta=\cos \zeta$. In view of the periodicity of the sinh function in the complex plane, we can restrict the possible values that the rapidities can take to the strip $-\pi / 2<\operatorname{Im} \lambda \leq \pi / 2$, or alternately define an extended zone scheme in which $\lambda$ and $\lambda+i \pi \mathbb{Z}$ are identified.

A more practical version of the Bethe equations is obtained by writing them in logarithmic form,

$$
\operatorname{atan}\left[\frac{\tanh \left(\lambda_{j}\right)}{\tan (\zeta / 2)}\right]-\frac{1}{M} \sum_{k=1}^{N} \operatorname{atan}\left[\frac{\tanh \left(\lambda_{j}-\lambda_{k}\right)}{\tan \zeta}\right]=\pi \frac{I_{j}}{M} .
$$

Here, $I_{j}$ are distinct half-integers which can be viewed as quantum numbers: each choice of a set $\left\{I_{j}\right\}, j=1, \ldots, N$ (with $I_{j}$ defined $\bmod (M)$ ) uniquely specifies a set of rapidities, and therefore an eigenstate. The energy of a state is given as a function of the rapidities by

$$
E=J \sum_{j=1}^{N} \frac{-\sin ^{2} \zeta}{\cosh 2 \lambda_{j}-\cos \zeta}-h\left(\frac{M}{2}-N\right),
$$

whereas the momentum has a simple representation in terms of the quantum numbers,

$$
q=\sum_{j=1}^{N} i \ln \left[\frac{\sinh \left(\lambda_{j}+i \zeta / 2\right)}{\sinh \left(\lambda_{j}-i \zeta / 2\right)}\right]=\pi N+\frac{2 \pi}{M} \sum_{j=1}^{N} I_{j} \quad \bmod 2 \pi
$$

The ground state is given by $I_{j}^{0}=-\frac{N+1}{2}+j, j=1, \ldots, N$, and all excited states are in principle obtained from the different choices of sets $\left\{I_{j}\right\}$.

To study dynamics, some ingredients have to be added to the Bethe Ansatz: the matrix elements of spin operators between eigenstates (form factors). In terms of form factors for the Fourier-transformed spin operators $S_{q}^{a}=\frac{1}{\sqrt{M}} \sum_{j=1}^{M} e^{i q j} S_{j}^{a}$, the structure factor can be written as a sum

$$
S^{a \bar{a}}(q, \omega)=2 \pi \sum_{\Psi \neq \Psi_{g}}\left|\left\langle\Psi_{g}\left|S_{q}^{a}\right| \Psi\right\rangle\right|^{2} \delta\left(\omega-\omega_{\Psi}\right)
$$

over the whole set of intermediate eigenstates $|\Psi\rangle$ (distinct from the ground state $\left|\Psi_{g}\right\rangle$ ) in a fixed magnetization subspace. Each term in (3.23) can be obtained [23] as a product of determinants of specific matrices, which are fully determined for given bra and ket eigenstates by a knowledge of the corresponding sets of rapidities. The analytical summation of this series remains for the moment out of reach, but numerically, for chains of length a few hundred sites, quite feasible. Moreover, we know that the correlation functions of the finite chain approach their thermodynamic limit with errors of order $\frac{1}{M}$, hence if $M=200$ for example the error is usually quite acceptable to make comparison with experiments. 
The strategy to follow is now clear. We compute the $S^{z z}$ and $S^{-+}$structure factors by directly summing the terms on the right-hand side of equation (3.23) over a judiciously chosen subset of eigenstates. The momentum delta functions are broadened to width $\varepsilon \sim 1 / M$ using $\delta_{\varepsilon}(x)=$ $\frac{1}{\sqrt{\pi} \varepsilon} e^{-x^{2} / \varepsilon^{2}}$ in order to obtain smooth curves. We scan through the eigenstates in the following order. First, we observe that the form factors of the spin operators between the ground state and an eigenstate $\{\lambda\}$ are extremely rapidly decreasing functions of the number of holes that need to be inserted in the configuration of the lowest-energy state (in the same base) in order to obtain the configuration $\{I\}$ corresponding to $\{\lambda\}$. We therefore scan through all bases and configurations for increasing number of holes, starting from one-hole states for $S^{z z}$, and zero-hole states for $S^{-+}$. Although the number of possible configurations for fixed base and number of holes is a rapidly increasing function of the number of holes, we find that the total contributions for fixed bases also rapidly decrease for increasing hole numbers. We therefore limit ourselves to states with up to three holes, corresponding to up to six-particle excitations. We can quantify the quality of the present computational method by evaluating the sum rules for the longitudinal and transverse form factors. Namely, by integrating over momentum and frequency, we should saturate the values

$$
\begin{gathered}
\int_{-\infty}^{\infty} \frac{d \omega}{2 \pi} \frac{1}{M} \sum_{q} S^{z z}(q, \omega)=\frac{1}{4}-\left\langle S^{z}\right\rangle^{2}=\frac{1}{4}\left[1-\left(1-\frac{2 N}{M}\right)^{2}\right] \\
\int_{-\infty}^{\infty} \frac{d \omega}{2 \pi} \frac{1}{M} \sum_{q} S^{-+}(q, \omega)=\frac{1}{2}-\left\langle S^{z}\right\rangle=\frac{N}{M} .
\end{gathered}
$$

For all intermediate states involving strings, we explicitly check that the deviations from the string hypothesis are small. We find in general that states involving strings of length higher that two are admissible solutions to the Bethe equations for high enough magnetizations. At zero field, only two-string states have exponentially small deviations $\delta$, and all higher-string states must be discarded.

The relative contributions to the structure factors from different bases is very much dependent on the system size, the anisotropy, and the magnetization. In general, we find that two- and four-particle contributions are sufficient to saturate well over $90 \%$ of the sum rules in all cases, for system sizes up to $M=200$. Interestingly, however, we find that string states also contribute noticeably in many cases. For example, the contribution to the zero-field transverse structure factor coming from intermediate states with one string of length two and up to three holes is of the order of six or seven percent of the weight, and similar or somewhat lower figures are found in other cases. Strings of length higher than two do not contribute significantly. The imperfect saturation of the sum rules that we obtain in general can be ascribed either to higher states in the hierarchy which are not included in our partial summations, or states that are in principle included, but which are rejected in view of their deviations from the string hypothesis. As the proportion of excluded string states can be rather large (ranging anywhere from zero to fifty percent), we believe the latter explanation to be the correct one. In any case, these results are precise enough to be compared successfully to different data from neutron scattering experiments for several magnetic compounds. From our results covering the whole Brillouin zone and frequency space, it is straightforward to obtain space-time dependent correlation functions by inverse Fourier transform:

$$
\left\langle S_{j+1}^{a}(t) S_{1}^{\bar{a}}(0)\right\rangle_{c}=\frac{1}{M} \sum_{\Psi \neq \Psi_{g}}\left|\left\langle\Psi_{g}\left|S_{q_{\Psi}}^{a}\right| \Psi\right\rangle\right|^{2} e^{-i q_{\Psi} j-i \omega_{\Psi} t}
$$


It is possible to compare these results to known exact results for equal-time correlation functions at short distance, and to the large-distance asymptotic form obtained from conformal field theory. This comparison can only be made at zero field, where both sets of results are known exactly. The comparison turns out to be extremely good, as can be expected from the high saturation of the sum rules [36].

\section{Correlation functions : infinite chain}

In the thermodynamic limit, $M \rightarrow \infty$ and at zero magnetic field, the model exhibits three different regimes depending on the value of $\Delta$ [3]. For $\Delta<-1$, the model is ferromagnetic, for $-1<\Delta<1$, the model has a non degenerated anti ferromagnetic ground state, and no gap in the spectrum (massless regime), while for $\Delta>1$, the ground state is twice degenerated with a gap in the spectrum (massive regime). In both cases, the ground state has spin zero. Hence the number of parameters $\lambda$ in the ground state vectors is equal to half the size $M$ of the chain. For $M \rightarrow \infty$, these parameters will be distributed in some continuous interval according to a density function $\rho$.

\subsection{The thermodynamic limit}

In this limit, the Bethe equations for the ground state, written in their logarithmic form, become a linear integral equation for the density distribution of these $\lambda$ 's,

$$
\rho_{\mathrm{tot}}(\alpha)+\int_{-\Lambda}^{\Lambda} K(\alpha-\beta) \rho_{\mathrm{tot}}(\beta) d \beta=\frac{p_{0_{\mathrm{tot}}}^{\prime}(\alpha)}{2 \pi},
$$

where the new real variables $\alpha$ are defined in terms of general spectral parameters $\lambda$ differently in the two domains. From now on, we only describe the massless regime (see [24] for the other case) $-1<\Delta<1$ where $\alpha=\lambda$. The density $\rho$ is defined as the limit of the quantity $\frac{1}{M\left(\lambda_{j+1}-\lambda_{j}\right)}$, and the functions $K(\lambda)$ and $p_{0_{\text {tot }}}^{\prime}(\lambda)$ are the derivatives with respect to $\lambda$ of the functions $-\frac{\theta(\lambda)}{2 \pi}$ and $p_{0_{\text {tot }}}(\lambda)$ :

$$
\begin{aligned}
& K(\alpha)=\frac{\sin 2 \zeta}{2 \pi \sinh (\alpha+i \zeta) \sinh (\alpha-i \zeta)} \quad \text { for }-1<\Delta<1, \text { with } \zeta=i \eta, \\
& p_{0}^{\prime}(\alpha)=\frac{\sin \zeta}{\sinh \left(\alpha+i \frac{\zeta}{2}\right) \sinh \left(\alpha-i \frac{\zeta}{2}\right)} \\
& \text { with } \quad p_{0_{\mathrm{tot}}}^{\prime}(\alpha)=\frac{1}{M} \sum_{i=1}^{M} p_{0}^{\prime}\left(\alpha-\beta_{k}-i \frac{\zeta}{2}\right),
\end{aligned}
$$

where $\beta_{k}=\xi_{k}$. The integration limit $\Lambda$ is equal to $+\infty$ for $-1<\Delta<1$. The solution for the equation (4.1) in the homogeneous model where all parameters $\xi_{k}$ are equal to $\eta / 2$, that is the density for the ground state of the Hamiltonian in the thermodynamic limit, is given by the following function [41]:

$$
\rho(\alpha)=\frac{1}{2 \zeta \cosh \left(\frac{\pi \alpha}{\zeta}\right)}
$$

For technical convenience, we will also use the function,

$$
\rho_{\mathrm{tot}}(\alpha)=\frac{1}{M} \sum_{i=1}^{M} \rho\left(\alpha-\beta_{k}-i \frac{\zeta}{2}\right)
$$


It will be also convenient to consider, without any loss of generality, that the inhomogeneity parameters are contained in the region $-\zeta<\operatorname{Im} \beta_{j}<0$. Using these results, for any $\mathscr{C}^{\infty}$ function $f\left(\pi\right.$-periodic in the domain $\Delta>1$ ), sums over all the values of $f$ at the point $\alpha_{j}, 1 \leq j \leq N$, parameterizing the ground state, can be replaced in the thermodynamic limit by an integral:

$$
\frac{1}{M} \sum_{j=1}^{N} f\left(\alpha_{j}\right)=\int_{-\Lambda}^{\Lambda} f(\alpha) \rho_{\mathrm{tot}}(\alpha) d \alpha+O\left(M^{-1}\right)
$$

Thus, multiple sums obtained in correlation functions will become multiple integrals. Similarly, it is possible to evaluate the behavior of the determinant formulas for the scalar products and the norm of Bethe vectors (and in particular their ratios) in the limit $M \rightarrow \infty$.

\subsection{Elementary blocks}

From the representations as multiple sums of these elementary blocks in the finite chain we can obtain their multiple integral representations in the thermodynamic limit. Let us now consider separately the two regimes of the $X X Z$ model. In the massless regime $\eta=-i \zeta$ is imaginary, the ground state parameters $\lambda$ are real and the limit of integration is infinity $\Lambda=\infty$. In this case we consider the inhomogeneity parameters $\xi_{j}$ such that $0>\operatorname{Im}\left(\xi_{j}\right)>-\zeta$. For the correlation functions in the thermodynamic limit one obtains the following result in this regime:

PROPOSITION 4.1.

$$
\begin{aligned}
& F_{m}\left(\left\{\varepsilon_{j}, \varepsilon_{j}^{\prime}\right\}\right)=\prod_{k<l} \frac{\sinh \frac{\pi}{\zeta}\left(\xi_{k}-\xi_{l}\right)}{\sinh \left(\xi_{k}-\xi_{l}\right)} \prod_{j=1}^{s^{\prime}} \int_{-\infty-i \zeta}^{\infty-i \zeta} \frac{d \lambda_{j}}{2 i \zeta} \prod_{j=s^{\prime}+1}^{m} \int_{-\infty}^{\infty} i \frac{d \lambda_{j}}{2 \zeta} \\
& \prod_{a=1}^{m} \prod_{k=1}^{m} \frac{1}{\sinh \frac{\pi}{\zeta}\left(\lambda_{a}-\xi_{k}\right)} \prod_{j \in \alpha^{-}}\left(\prod_{k=1}^{j-1} \sinh \left(\mu_{j}-\xi_{k}-i \zeta\right) \prod_{k=j+1}^{m} \sinh \left(\mu_{j}-\xi_{k}\right)\right) \\
& \prod_{j \in \alpha^{+}}\left(\prod_{k=1}^{j-1} \sinh \left(\mu_{j}^{\prime}-\xi_{k}+i \zeta\right) \prod_{k=j+1}^{m} \sinh \left(\mu_{j}^{\prime}-\xi_{k}\right)\right) \prod_{a>b} \frac{\sinh \frac{\pi}{\zeta}\left(\lambda_{a}-\lambda_{b}\right)}{\sinh \left(\lambda_{a}-\lambda_{b}-i \zeta\right)},
\end{aligned}
$$

with the parameters of integration ordered in the following way

$$
\left\{\lambda_{1}, \ldots \lambda_{m}\right\}=\left\{\mu_{j_{\max }^{\prime}}^{\prime}, \ldots, \mu_{j_{\min }^{\prime}}^{\prime}, \mu_{j_{\min }}, \ldots, \mu_{j_{\max }}\right\} .
$$

The homogeneous limit $\left(\xi_{j}=-i \zeta / 2, \forall j\right)$ of the correlation function $F_{m}\left(\left\{\varepsilon_{j}, \varepsilon_{j}^{\prime}\right\}\right)$ can then be taken in an obvious way. We have obtained similar representations for the massive regime, and also in the presence of a non-zero magnetic field [24]. For zero magnetic field, these results agree exactly with the ones obtained by Jimbo and Miwa in [55], using in particular $q$-KZ equations. It means that for zero magnetic field, the elementary blocks of correlation functions indeed satisfy $q-\mathrm{KZ}$ equations. Recently, more algebraic representations of solutions of the $q$-KZ equations have been obtained that correspond to the above correlation functions [56, 57, 58, 59]. From the finite chain representation for the two-point function, it is also possible to obtain multiple integral representations for that case as well, in particular for their generating function [26, 28]. They correspond different huge re-summations and symmetrization of the corresponding elementary blocks, as in the finite chain situation [26]. Moreover, the case of time dependent correlation functions as also been obtained [30,31]. Finally, let us note that at the free fermion point, all the results presented here lead, in a very elementary way, to already know results [27, 32, 34]. 


\section{Exact and asymptotic results}

\subsection{Exact results at $\Delta=1 / 2$}

Up to now, two exact results have been obtained for the case of anisotropy $\Delta=1 / 2:$ the exact value of the emptiness formation probability for arbitrary distance $m$ [28] and the two point function of the third component of spin [33]. These two results follow from the above multiple integral representations for which, due to the determinant structure of the integrand, the corresponding multiple integrals can be separated and hence explicitly computed for this special value of the anisotropy.

\subsubsection{The emptiness formation probability}

This correlation function $\tau(m)$ (the probability to find in the ground state a ferromagnetic string of length $m$ ) is defined as the following expectation value

$$
\tau(m)=\left\langle\psi_{g}\left|\prod_{k=1}^{m} \frac{1-\sigma_{k}^{z}}{2}\right| \psi_{g}\right\rangle
$$

where $\left|\psi_{g}\right\rangle$ denotes the normalized ground state. In the thermodynamic limit $(M \rightarrow \infty)$, this quantity can be expressed as a multiple integral with $m$ integrations [54, 55, 8, 23, 24].

Proposition 5.1. For $\Delta=\cos \zeta, 0<\zeta<\pi, \tau(m)=\lim _{\xi_{1}, \ldots \xi_{m} \rightarrow-\frac{i \zeta}{2}} \tau\left(m,\left\{\xi_{j}\right\}\right)$, where

$$
\begin{gathered}
\tau\left(m,\left\{\xi_{j}\right\}\right)=\frac{1}{m !} \int_{-\infty}^{\infty} \frac{Z_{m}(\{\lambda\},\{\xi\})}{\prod_{a<b}^{m} \sinh \left(\xi_{a}-\xi_{b}\right)} \operatorname{det}_{m}\left(\frac{i}{2 \zeta \sinh \frac{\pi}{\zeta}\left(\lambda_{j}-\xi_{k}\right)}\right) d^{m} \lambda, \\
Z_{m}(\{\lambda\},\{\xi\})=\prod_{a=1}^{m} \prod_{b=1}^{m} \frac{\sinh \left(\lambda_{a}-\xi_{b}\right) \sinh \left(\lambda_{a}-\xi_{b}-i \zeta\right)}{\sinh \left(\lambda_{a}-\lambda_{b}-i \zeta\right)} . \\
\cdot \frac{\operatorname{det}_{m}\left(\frac{-i \sin \zeta}{\sinh \left(\lambda_{j}-\xi_{k}\right) \sinh \left(\lambda_{j}-\xi_{k}-i \zeta\right)}\right)}{\prod_{a>b}^{m} \sinh \left(\xi_{a}-\xi_{b}\right)} .
\end{gathered}
$$

The proof is given in [26]. Due to the determinant structure of the integrand, the integrals can be separated and computed for the special case $\Delta=\frac{1}{2}(\zeta=\pi / 3)$ :

PROPOSITION 5.2. Let $\xi_{k}=\varepsilon_{k}-i \pi / 6$ and $\varepsilon_{a b}=\varepsilon_{a}-\varepsilon_{b}$, we obtain,

$$
\begin{aligned}
& \tau\left(m,\left\{\varepsilon_{j}\right\}\right)=\frac{(-1)^{\frac{m^{2}-m}{2}}}{2^{m^{2}}} \prod_{a>b}^{m} \frac{\sinh 3 \varepsilon_{b a}}{\sinh \varepsilon_{b a}} \prod_{\substack{a, b=1 \\
a \neq b}}^{m} \frac{1}{\sinh \varepsilon_{a b}} \cdot \operatorname{det}_{m}\left(\frac{3 \sinh \frac{\varepsilon_{j k}}{2}}{\sinh \frac{3 \varepsilon_{j k}}{2}}\right), \\
& \tau(m)=\left(\frac{1}{2}\right)^{m^{2}} \prod_{k=0}^{m-1} \frac{(3 k+1) !}{(m+k) !}
\end{aligned}
$$

Observe that the quantity $A_{m}=\prod_{k=0}^{m-1}(3 k+1) ! /(m+k)$ ! is the number of alternating sign matrices of size $m$. This result was conjectured in [67]. 


\subsubsection{The two point function of $\sigma^{z}$}

The two point functions can be obtained, as in the finite chain situation, from a generating function $\left\langle Q_{\kappa}(m)\right\rangle$; in the thermodynamic limit, we use the following multiple integral representation [33]:

$$
\begin{gathered}
\left\langle Q_{\kappa}(m)\right\rangle=\sum_{n=0}^{m} \frac{\kappa^{m-n}}{n !(m-n) !} \oint_{\Gamma\{-i \zeta / 2\}} \frac{d^{m} z}{(2 \pi i)^{m}} \int_{\mathbb{R}-i \zeta} d^{n} \lambda \int_{\mathbb{R}} d^{m-n} \lambda \cdot \prod_{j=1}^{m} \frac{\varphi^{m}\left(z_{j}\right)}{\varphi^{m}\left(\lambda_{j}\right)} \\
\prod_{j=1}^{n}\left\{t\left(z_{j}, \lambda_{j}\right) \prod_{k=1}^{m} \frac{\sinh \left(z_{j}-\lambda_{k}-i \zeta\right)}{\sinh \left(z_{j}-z_{k}-i \zeta\right)}\right\} \prod_{j=n+1}^{m}\left\{t\left(\lambda_{j}, z_{j}\right) \prod_{k=1}^{m} \frac{\sinh \left(\lambda_{k}-z_{j}-i \zeta\right)}{\sinh \left(z_{k}-z_{j}-i \zeta\right)}\right\} \\
\prod_{j=1}^{m} \prod_{k=1}^{m} \frac{\sinh \left(\lambda_{k}-z_{j}-i \zeta\right)}{\sinh \left(\lambda_{k}-\lambda_{j}-i \zeta\right)} \cdot \operatorname{det}_{m}\left(\frac{i}{2 \zeta \sinh \frac{\pi}{\zeta}(\lambda-z)}\right) .
\end{gathered}
$$

Here,

$$
\Delta=\cos \zeta, \quad t(z, \lambda)=\frac{-i \sin \zeta}{\sinh (z-\lambda) \sinh (z-\lambda-i \zeta)}, \quad \varphi(z)=\frac{\sinh \left(z-i \frac{\zeta}{2}\right)}{\sinh \left(z+i \frac{\zeta}{2}\right)},
$$

and the integrals over the variables $z_{j}$ are taken with respect to a closed contour $\Gamma$ which surrounds the point $-i \zeta / 2$ and does not contain any other singularities of the integrand. The equation (5.6) is valid for the homogeneous $X X Z$ chain with arbitrary $-1<\Delta<1$. If we consider the inhomogeneous $X X Z$ model with inhomogeneities $\xi_{1}, \ldots, \xi_{m}$, then one should replace in the representation (5.6) the function $\varphi^{m}$ in the following way:

$$
\varphi^{m}(z) \rightarrow \prod_{b=1}^{m} \frac{\sinh \left(z-\xi_{b}-i \zeta\right)}{\sinh \left(z-\xi_{b}\right)}, \quad \varphi^{-m}(\lambda) \rightarrow \prod_{b=1}^{m} \frac{\sinh \left(\lambda-\xi_{b}\right)}{\sinh \left(\lambda-\xi_{b}-i \zeta\right)} .
$$

In order to come back to the homogeneous case, one should set $\xi_{k}=-i \zeta / 2, k=1, \ldots, m$ in (5.8). In the inhomogeneous model, the integration contour $\Gamma$ surrounds the points $\xi_{1}, \ldots, \xi_{m}$, and the integrals over $z_{j}$ are therefore equal to the sum of the residues of the integrand in these simple poles. It turns out that again for the special case $\Delta=\frac{1}{2}$ integrals can be separated and computed to give :

PROPOSITION 5.3.

$$
\begin{aligned}
& \left\langle Q_{\kappa}(m)\right\rangle=\frac{3^{m}}{2^{m^{2}}} \prod_{a>b}^{m} \frac{\sinh 3\left(\xi_{a}-\xi_{b}\right)}{\sinh ^{3}\left(\xi_{a}-\xi_{b}\right)} \sum_{n=0}^{m} \kappa^{m-n} \sum_{\substack{\{\xi\}=\left\{\xi_{\gamma_{+}}\right\} \cup\left\{\xi_{\gamma_{-}}\right\} \\
\left|\gamma_{+}\right|=n}} \operatorname{det} \hat{\Phi}^{(n)} \\
& \times \prod_{a \in \gamma_{+}} \prod_{b \in \gamma_{-}} \frac{\sinh \left(\xi_{b}-\xi_{a}-\frac{i \pi}{3}\right) \sinh \left(\xi_{a}-\xi_{b}\right)}{\sinh ^{2}\left(\xi_{b}-\xi_{a}+\frac{i \pi}{3}\right)}, \\
& \hat{\Phi}^{(n)}\left(\left\{\xi_{\gamma_{+}}\right\},\left\{\xi_{\gamma_{-}}\right\}\right)=\left(\begin{array}{c|c}
\Phi\left(\xi_{j}-\xi_{k}\right) & \Phi\left(\xi_{j}-\xi_{k}-\frac{i \pi}{3}\right) \\
\hline \Phi\left(\xi_{j}-\xi_{k}+\frac{i \pi}{3}\right) & \Phi\left(\xi_{j}-\xi_{k}\right)
\end{array}\right), \Phi(x)=\frac{\sinh \frac{x}{2}}{\sinh \frac{3 x}{2}}
\end{aligned}
$$


Here the sum is taken with respect to all partitions of the set $\{\xi\}$ into two disjoint subsets $\left\{\xi_{\gamma_{+}}\right\} \cup$ $\left\{\xi_{\gamma_{-}}\right\}$of cardinality $n$ and $m-n$ respectively. The first $n$ lines and columns of the matrix $\hat{\Phi}^{(n)}$ are associated with the parameters $\xi \in\left\{\xi_{\gamma_{+}}\right\}$. The remaining lines and columns are associated with $\xi \in\left\{\xi_{\gamma_{-}}\right\}$.

Thus, we have obtained an explicit answer for the generating function $\left\langle Q_{\kappa}(m)\right\rangle$ of the inhomogeneous $X X Z$ model. It is also possible to check that the above sum over partitions remains indeed finite in the homogeneous limit $\xi_{k} \rightarrow 0$.

\subsection{Asymptotic results}

An important issue is the analysis of the multiple integral representations of correlation functions for large distances. There it means analyzing asymptotic behavior of $m$-fold integrals for $m$ large. An interesting example to study in this respect is provided by the emptiness formation probability. This correlation function reduces to a single elementary block. Moreover, we already described its exact value for an anisotropy $\Delta=\frac{1}{2}$ in the previous section. In fact, it is possible to obtain the asymptotic behavior of $\tau(m)$ using the saddle-point method for arbitrary values of the anisotropy $\Delta>-1$. This was performed for the first time in [27] in the case of free fermions $(\Delta=0)$, but it can be applied to the general case as well. We present here the results in the massless and massive regimes $[29,34]$.

To apply the saddle-point method to the emptiness formation probability, it is convenient to express its integral representation in the following form:

$$
\tau(m)=\int_{\mathscr{D}} d^{m} \lambda G_{m}(\{\lambda\}) e^{m^{2} S_{m}(\{\lambda\})},
$$

with

$$
\begin{aligned}
S_{m}(\{\lambda\})= & -\frac{1}{m^{2}} \sum_{a>b}^{m} \log \left[\sinh \left(\lambda_{a}-\lambda_{b}+\eta\right) \sinh \left(\lambda_{a}-\lambda_{b}-\eta\right)\right] \\
& +\frac{1}{m} \sum_{a=1}^{m} \log \left[\sinh \left(\lambda_{a}+\eta / 2\right) \sinh \left(\lambda_{a}-\eta / 2\right)\right] \\
& +\frac{1}{m^{2}} \lim _{\xi_{1} \ldots \xi_{m} \rightarrow \eta / 2} \log \left[\left(\frac{-2 i \pi}{\sinh \eta}\right)^{m} \frac{\left(\operatorname{det} \rho\left(\lambda_{j}, \xi_{k}\right)\right)^{2}}{\prod_{a \neq b} \sinh \left(\xi_{a}-\xi_{b}\right)}\right]
\end{aligned}
$$

and

$$
G_{m}(\{\lambda\})=\lim _{\xi_{1} \ldots \xi_{m} \rightarrow \eta / 2} \frac{\operatorname{det}_{m}\left[\frac{i}{2 \pi} t\left(\lambda_{j}, \xi_{k}\right)\right]}{\operatorname{det}_{m} \rho\left(\lambda_{j}, \xi_{k}\right)} .
$$

In (5.10), the integration domain $\mathscr{D}$ is such that the variable of integration $\lambda_{1}, \ldots, \lambda_{m}$ are ordered in the interval $\mathscr{C}=\left[-\Lambda_{h}, \Lambda_{h}\right]$ (i.e. $-\Lambda_{h}<\lambda_{1}<\cdots<\lambda_{m}<\Lambda_{h}$ in the massless case, and $-i \Lambda_{h}<$ $i \lambda_{1}<\cdots<i \lambda_{m}<i \Lambda_{h}$ in the massive case).

The main problem in the saddle point analysis is that, a priori, we do not know any asymptotic equivalent of the quantity $G_{m}(\lambda)$ when $m \rightarrow \infty$. Nevertheless, in the case of zero magnetic field, it is still possible to compute the asymptotic behavior of (5.10) in the leading order, provided we make the following hypothesis: we assume that the integrand of (5.10) admits a maximum for a 
certain value $\lambda_{1}^{\prime}, \ldots, \lambda_{m}^{\prime}$ of the integration variables $\lambda_{1}, \ldots, \lambda_{m}$, that, for large $m$, the distribution of these parameters $\lambda_{1}^{\prime}, \ldots, \lambda_{m}^{\prime}$ can be described by a density function $\rho_{s}\left(\lambda^{\prime}\right)$ of the form

$$
\rho_{s}\left(\lambda_{j}^{\prime}\right)=\lim _{m \rightarrow \infty} \frac{1}{m\left(\lambda_{j+1}^{\prime}-\lambda_{j}^{\prime}\right)},
$$

on the symmetric interval $[-\Lambda, \Lambda]$ and that, at the leading order in $m$, we can replace the sums over the set of parameters $\left\{\lambda^{\prime}\right\}$ by integrals weighted with the density $\rho_{s}\left(\lambda^{\prime}\right)$.

First, it is easy to determine the maximum of the function $S_{m}(\{\lambda\})$. Indeed, let $\{\tilde{\lambda}\}$ be solution of the system

$$
\partial_{\lambda_{j}} S_{m}(\{\tilde{\lambda}\})=0, \quad 1 \leq j \leq m .
$$

In the limit $m \rightarrow \infty$, if we suppose again that the parameters $\tilde{\lambda}_{1}, \ldots, \tilde{\lambda}_{m}$ become distributed according to a certain density $\tilde{\rho}_{s}(\lambda)$ and that sums over the $\tilde{\lambda}_{j}$ become integrals over this density, the system (5.14) turns again into a single integral equation for $\tilde{\rho}_{s}$, that can be solved explicitly in the case of zero magnetic field. It gives the maximum of $S_{m}(\{\lambda\})$ when $m \rightarrow \infty^{2}$.

The second step is to show that the factor $G_{m}(\{\lambda\})$ gives always a negligible contribution compared to $S_{m}(\{\tilde{\lambda}\})$ at this order in $m$, at least for any distribution of the variables $\lambda_{j}$ satisfying the previous hypothesis of regularity. We obtain,

$$
\lim _{m \rightarrow \infty} \frac{1}{m^{2}} \log G_{m}(\{\lambda\})=0
$$

for any distribution of $\{\lambda\}$ with good properties of regularity, in particular for the saddle point. This means that, at the main order in $m$, the factor $G_{m}(\{\lambda\})$ does not contribute to the value of the maximum of the integrand.

Finally we obtain the following result concerning the asymptotic behaviour of $\tau(m)$ for $m \rightarrow \infty$ (see $[29,34])$ :

$$
\begin{array}{rlrl}
S^{(0)}(\Delta) & =\lim _{m \rightarrow \infty} \frac{\log \tau(m)}{m^{2}}, \\
& =-\frac{\zeta}{2}-\sum_{n=1}^{\infty} \frac{e^{-n \zeta}}{n} \frac{\sinh (n \zeta)}{\cosh (2 n \zeta)}, & (\Delta=\cosh \zeta>1), \\
& =\log \frac{\pi}{\zeta}+\frac{1}{2} \int_{\mathbb{R}-i 0} \frac{d \omega}{\omega} \frac{\sinh \frac{\omega}{2}(\pi-\zeta) \cosh ^{2} \frac{\omega \zeta}{2}}{\sinh \frac{\pi \omega}{2} \sinh \frac{\omega \zeta}{2} \cosh \omega \zeta}, & (-1<\Delta=\cos \zeta<1) .
\end{array}
$$

It coincides with the exact known results obtained in $[68,27]$ at the free fermion point and in $[67,28]$ at $\Delta=1 / 2$, and is in agreement with the expected (infinite) value in the Ising limit. Similar techniques can be applied to the two point function. However, the result that has been extracted so far is only the absence of the gaussian term. Unfortunately, we do not know up to now how to extract the expected power law corrections to the gaussian behavior from this saddle point analysis. More powerful methods will certainly be needed to go further.

\footnotetext{
${ }^{2}$ At this main order in $m$, there exists a unique solution of the integral equation for $\tilde{\rho}_{s}$, and we know it corresponds to a maximum because $S_{m}(\{\lambda\}) \rightarrow-\infty$ on the boundary of $\mathscr{D}$.
} 


\section{Conclusion and perspectives}

In this article, we have reviewed recent results concerning the computation of correlation functions in the $X X Z$ chain by the methods of the inverse scattering problem and the algebraic Bethe ansatz. In conclusion, we would like to discuss some perspectives and problems to be solved.

One of the most interesting open problems is to prove the conformal field theory predictions $[69,70]$ concerning the asymptotic behavior of the correlation functions. This is certainly a very important issue not only for physical applications but also from a theoretical view point. Moreover, it also would open the route towards the generalization of the methods presented here to quantum integrable models of field theory. We have seen that in particular cases, the multiple integral representations enable for a preliminary asymptotic analysis . Nevertheless, this problem remains one of the main challenges in the topics that have been described in this article.

A possible way to solve this problem would be to find the thermodynamic limit of the master equations (like the one obtained for the two point correlation functions). It is natural to expect that, in this limit, one should obtain a representation for these correlation functions in terms of a functional integral, which could eventually be estimated for large time and distance.

Note that the master equation shows a direct analytic relation between the multiple integral representations and the form factor expansions for the correlation functions. It seems likely that similar representations exist for other models solvable by algebraic Bethe ansatz. It would be in particular very interesting to obtain an analogue of this master equation in the case of the field theory models, which could provide an analytic link between short distance and long distance expansions of their correlation functions. Preliminary results in this direction have been obtained recently in [71] for the quantum non-linear Schroedinger model.

From the condensed matter view point there is also quite some interest in the extension of the results presented here for different other models. It includes spin chains with impurities [72] or with open boundaries [73, 74], and also electronic models like the Hubbard model for which progress in the understanding of the space of states are necessary to apply our method.

\section{Acknowledgements}

It is a great pleasure to thank N. Kitanine, N. A. Slavnov and V. Terras for their longstanding collaboration on the difficult but exiting topics presented here, and J.-S. Caux and R. Hagemans for their energy and skills that make our collaboration successful in comparing Bethe ansatz results to actual neutron scattering experiments.

\section{References}

[1] H. Bethe, Zur Theorie der Metalle I. Eigenwerte und Eigenfunktionen Atomkete, Z. Phys. 71 (1931), 205.

[2] W. Heisenberg, Zur Theorie der Ferromagnetismus, Z. Phys. 49 (1928), 619.

[3] R. J. Baxter, Exactly solved models in statistical mechanics (Academic Press, London - New York, 1982).

[4] M. Gaudin, La fonction d'onde de Bethe (Masson, 1983). 
[5] E. H. Lieb and D. C. Mattis, Mathematical Physics in One Dimension (Academic Press, New-York, 1966).

[6] H. B. Thacker, Exact integrability in quantum field theory and statistical systems, Rev. Mod. Phys. 53 (1981), 253.

[7] V. E. Korepin, N. M. Bogoliubov, and A. G. Izergin, Quantum inverse scattering method and correlation functions (Cambridge University Press, 1993).

[8] M. Jimbo and T. Miwa, Algebraic analysis of solvable lattice models (AMS, 1995).

[9] L. D. Faddeev, E. K. Sklyanin, and L. A. Takhtajan, Quantum inverse problem method I, Theor. Math. Phys. 40 (1980), 688.

[10] L. A. Takhtajan and L. D. Faddeev, The Quantum method of the inverse problem and the Heisenberg XYZ model, Russ. Math. Surveys 34 (1979), 11.

[11] P. P. Kulish, N. Yu. Reshetikhin, and E. K. Sklyanin, Yang-Baxter equation and representation theory I, Lett. Math. Phys., 5 393-403, (1981).

[12] P. P. Kulish and N. Yu. Reshetikhin, Quantum linear problem for the sine-Gordon equation and higher representations, Zap. Nauch. Sem. LOMI, 101:101-110, (1981), Translation in J. Sov. Math. 23, 2435-41 (1983).

[13] P. P. Kulish and N. Yu. Reshetikhin, $G_{3}$-invariant solutions of the Yang-Baxter equation and associated quantum systems, J. Sov. Math., 34:1948-1971, (1986), translated from Zap. Nauch. Sem. LOMI 120, 92-121 (1982).

[14] P. P. Kulish and E. K. Sklyanin, Quantum spectral transform method. Recent developments, Lectures Notes in Physics, 151:61-119, (1982).

[15] M. Jimbo, A $q$-difference analogue of $U(g)$ and the Yang-Baxter equation, Lett. Math. Phys., 10, 63-69 (1985).

[16] M. Jimbo, A $q$-analogue of $U(g l(N+1))$, Hecke algebra, and the Yang-Baxter equation, Lett. Math. Phys., 11, 247-252 (1986).

[17] V. G. Drinfeld, Hopf algebras and the quantum Yang-Baxter equation, Soviet Math. Dokl., 32(1), 254-258 (1985).

[18] V. G. Drinfel'd, Quantum groups, Proceedings of the ICM 1986, Berkeley, USA, 1986, AMS, (1987).

[19] E. K. Sklyanin The quantum Toda chain Lecture Notes in Physics 226, 196-233 (1985).

[20] E. K. Sklyanin Separation of variables - New trends Prog. Theor. phys. suppl. 118, 35-60 (1995).

[21] V. B. Kuznetsov, V. V. Mangazeev, E. K. Sklyanin Q-operator and factorised separation chain for Jack polynomials Ind. Math. New series 14, 451-482 (2003).

[22] J. M. Maillet, and J. Sanchez de Santos, Drinfeld twists and algebraic Bethe ansatz, Amer. Math. Soc. Transl. 201 (2000), 137.

[23] N. Kitanine, J. M. Maillet, and V. Terras, Form factors of the XXZ Heisenberg spin-1/2 finite chain, Nucl. Phys. B 554 (1999), 647.

[24] N. Kitanine, J. M. Maillet, and V. Terras, Correlation functions of the XXZ Heisenberg spin-1/2 chain in a magnetic field, Nucl. Phys. B 567 (2000), 554.

[25] J. M. Maillet and V. Terras, On the quantum inverse scattering problem, Nucl. Phys. B 575 (2000), 627.

[26] N. Kitanine, J. M. Maillet, N. A. Slavnov, and V. Terras, Spin-spin correlation functions of the XXZ-1/2 Heisenberg chain in a magnetic field, Nucl. Phys. B 641 (2002), 487. 
[27] N. Kitanine, J. M. Maillet, N. A. Slavnov, and V. Terras, Correlation functions of the XXZ spin-1/2 Heisenberg chain at the free fermion point from their multiple integral representations, Nucl. Phys. B 642 (2002), 433.

[28] N. Kitanine, J. M. Maillet, N. A. Slavnov, and V. Terras, Emptiness formation probability of the XXZ spin-1/2 Heisenberg chain at Delta = 1/2, J.Phys. A 35 (2002), L385.

[29] N. Kitanine, J. M. Maillet, N. A. Slavnov, and V. Terras, Large distance asymptotic behaviour of the emptiness formation probability of the XXZ spin-1/2 Heisenberg chain, J.Phys. A 35 (2002), L753.

[30] N. Kitanine, J. M. Maillet, N. A. Slavnov, and V. Terras, Master equation for spin-spin correlation functions of the XXZ chain, Nucl. Phys. B 712 [FS] (2005), 600.

[31] N. Kitanine, J. M. Maillet, N. A. Slavnov, and V. Terras, Dynamical correlation functions of the XXZ spin-1/2 chain, Nucl. Phys. B 729 [FS] (2005), 558.

[32] N. Kitanine, J. M. Maillet, N. A. Slavnov, and V. Terras, On the spin-spin correlation functions of the XXZ spin-1/2 infinite chain, J.Phys. A 38 (2005), 7441.

[33] N. Kitanine, J. M. Maillet, N. A. Slavnov, and V. Terras, Exact results for the sigma-z two-point function of the XXZ chain at Delta=1/2, J. Stat. Mech. (2005) L09002.

[34] N. Kitanine, J. M. Maillet, N. A. Slavnov, and V. Terras, On the algebraic Bethe Ansatz approach to the correlation functions of the XXZ spin-1/2 Heisenberg chain In Recent Progress in Solvable lattice Models, RIMS Sciences Project Research 2004 on Method of Algebraic Analysis in Integrable Systems, RIMS, Kyoto, Kokyuroku, 1480, 14 (2006); hep-th/0505006.

[35] J.-S. Caux and J. M. Maillet, Computation of dynamical correlation functions of Heisenberg chains in a field, Phys. Rev. Lett. 95, 077201 (2005).

[36] J.-S. Caux, R. Hagemans, and J. M. Maillet, Computation of dynamical correlation functions of Heisenberg chains: the gapless anysotropic regime, J. Stat. Mech., P09003 (2005).

[37] R. G. Pereira, J. Sirker, J.-S. Caux, R. Hagemans, J. M. Maillet, S. R. White, and I. Affleck, Dynamical Spin Structure Factor for the Anisotropic Spin-1/2 Heisenberg Chain Phys. Rev. Lett. 96, 257202 (2006).

[38] L. Hulthen, Über das Austauschproblem eines Kristalls, Arkiv. Mat. Astron. Fys., 26 A(11):1-106, 1938.

[39] R. Orbach, Linear antiferromagnetic chain with anisotropic coupling, Phys. Rev. 112 (1958), 309.

[40] L. R. Walker, Antiferromagnetic linear chain, Phys. Rev. 116 (1959), 1089.

[41] C. N. Yang and C. P. Yang, One-dimensional chain of anisotropic spin-spin interactions. I. Proof of Bethe's hypothesis for ground state in a finite system, Phys. Rev. 150 (1966), 321.

[42] C. N. Yang and C. P. Yang, One-dimensional chain of anisotropic spin-spin interactions. II. Properties of the ground state energy per lattice site for an infinite system, Phys. Rev. 150 (1966), 327.

[43] L. Onsager, Cristal statistics I. A two-dimensional model with an order-disorder transition, Phys. Rev. 65 (1944), 117.

[44] B. Kaufmann Cristal statistics II. Partition function evaluated by spinor analysis, Phys. Rev. 76, 1232-1243 (1949).

[45] B. Kaufmann, L. Onsager, Cristal statistics III. Short range order in a binary Ising lattice, Phys. Rev. 76, 1244-1252 (1949).

[46] C. N. Yang, The spontaneous magnetization of a two-dimensional Ising model Phys. Rev. 85, 808-816 (1952). 
[47] E. Lieb, T. Schultz, and D. Mattis, Two soluble models of an antiferromagnetic chain, Ann. Phys. 16 (1961), 407.

[48] B. McCoy, Spin correlation functions of the XY model Phys. Rev. 173 (1968), 531.

[49] T. T. Wu, B. M. McCoy, C. A. Tracy, and E. Barouch, The spin-spin correlation function of the two-dimensional Ising model: exact results in the scaling region, Phys. Rev. B 13 (1976), 316.

[50] B. M. McCoy, C. A. Tracy, and T. T. Wu, Two-dimensional Ising model as an exactly soluble relativistic quantum field theory: explicit formulas for the $n$-point functions, Phys. Rev. Lett. 38 (1977), 793.

[51] M. Sato, T. Miwa, and M. Jimbo, Holonomic quantum fields, Publ. Res. Inst. Math. Sci. 14 (1978), 223; 15 (1979), 201, 577, 871; 16 (1980), 531.

[52] A. G. Izergin and V. E. Korepin, The quantum inverse scattering method approach to correlation functions, Com. Math. Phys. 94, 67-92 (1984).

[53] A. G. Izergin and V. E. Korepin, Correlation functions for the Heisenberg XXZ-antiferromagnet, Com. Math. Phys. 99, 271-302 (1985).

[54] M. Jimbo, K. Miki, T. Miwa, and A. Nakayashiki, Correlation functions of the XXZ model for $\Delta<-1$, Phys. Lett. A 168 (1992), 256.

[55] M. Jimbo and T. Miwa, Quantum KZ equation with $|q|=1$ and correlation functions of the XXZ model in the gapless regime, J. Phys. A. 29 (1996), 2923.

[56] H. Boos, M. Jimbo, T. Miwa, F. Smirnov, and Y. Takeyama, Reduced $q$-KZ equation and correlation functions of the XXZ model, Com. Math. Phys. 261, 245-276 (2006).

[57] H. Boos, M. Jimbo, T. Miwa, F. Smirnov, and Y. Takeyama, Density matrix of a finite sub-chain of the Heisenberg anti-ferromagnet Lett. Math. Phys. 75, 201-208 (2006).

[58] H. Boos, M. Jimbo, T. Miwa, F. Smirnov, and Y. Takeyama, Algebraic representation of correlation functions in integrable spin chains Ann. Hen. Poin. 7, 1395-1428 (2006).

[59] H. Boos, M. Jimbo, T. Miwa, F. Smirnov, and Y. Takeyama, Hidden grassmann structure in the XXZ model Com. Math. Phys. 272, 263-281 (2007).

[60] F. Göhmann, A. Klümper, and A. Seel, Integral representations for correlation functions of the XXZ chain at finite temperature, J. Phys. A 37 (2004), 7625.

[61] D. A. Tennant, R. A. Cowley, S. E. Nagler, and A. M. Tsvelik, Measurement of the spin-excitation continuum in one-dimensional KCuF3 using neutron scattering, Phys. Rev. B 52, 13368-13380 (1995).

[62] V. Tarasov and A. Varchenko, Completeness of Bethe vectors and difference equations with regular singular points, Int.Math.Res.Notices 13 (1996), 637.

[63] A. G. Izergin, Partition function of the six-vertex model in a finite volume, Sov. Phys. Dokl. 32 (1987), 878 .

[64] N. A. Slavnov, Calculation of scalar products of wave functions and form factors in the framework of the algebraic Bethe Ansatz, Theor. Math. Phys. 79 (1989), 502.

[65] M. Gaudin, B. M. Mc Coy and T. T. Wu, Normalization sum for the Bethe's hypothesis wave functions of the Heisenberg-Ising model, Phys. Rev. D 23 (1981), 417.

[66] V. E. Korepin, Calculation of norms of Bethe wave functions, Commun. Math. Phys. 86 (1982), 391.

[67] A. V. Razumov and Y. G. Stroganov, Spin chains and combinatorics, J. Phys. A 34, 3185 (2001). 
[68] M. Shiroishi, M. Takahashi, and Y. Nishiyama, Emptiness Formation Probability for the One-Dimensional Isotropic XY Model, J. Phys. Soc. Jap. 70, 3535 (2001).

[69] A. Luther and I. Peschel, Calculation of critical exponents in two dimensions from quantum field theory in one dimension, Phys. Rev. B 12, 3908 (1975).

[70] A. A. Belavin, A. M. Polyakov, and A. B. Zamolodchikov, Infinite conformal symmetry in two-dimensional quantum field theory, Nucl. Phys. B 241, 333 (1984).

[71] N. Kitanine, K. Kozlowski, J. M. Maillet, N. A. Slavnov, and V. Terras, On correlation functions of integrable models associated with the six-vertex R-matrix, J. Stat. Mech. P01022 (2007).

[72] O. Castro-Alvaredo and J. M. Maillet, Form factors of integrable Heisenberg (higher) spin chains to appear in J. Phys. A (2007), hep-th/0702186.

[73] N. Kitanine, K. Kozlowski, J. M. Maillet, G. Niccoli, N. A. Slavnov, and V. Terras, Correlation functions of the open XXZ chain I (To appear)

[74] N. Kitanine, K. Kozlowski, J. M. Maillet, G. Niccoli, N. A. Slavnov, and V. Terras, Correlation functions of the open XXZ chain II (To appear) 